\title{
REVESCO. Revista de Estudios Cooperativos
}

ISSN: $1885-8031$

http://dx.doi.org/10.5209/REVE.54925

\section{Las cooperativas de crédito andaluzas en el siglo $\mathrm{XX}$ : origen $\mathrm{y}$ consolidación del sector}

\author{
María José Vargas-Machuca Salido ${ }^{1}$
}

Recibido: 2 de noviembre de 2016 / Aceptado: 28 de diciembre de 2016

Resumen. El principal objetivo de este artículo es analizar la evolución de las cooperativas de crédito andaluzas durante el siglo XX, estudiando el desarrollo dese su origen hasta la consolidación del sector en la década de los noventa. Las primeras entidades de este tipo en Andalucía surgen en los primeros años de esa centuria, con algo de retraso respecto a otros países europeos y con menos impulso que en otras zonas de España. A partir de entonces, se crean numerosas entidades de pequeña dimensión vinculadas, en su mayoría a Sindicatos Agrarios o como secciones de crédito de cooperativas agrícolas que no llegarían a adquirir la condición de entidad de crédito.

Desde mediados de siglo, comienza a configurarse el sector andaluz de cajas rurales con instituciones que, en su mayoría, se mantienen operativas hasta finales de la centuria. El estudio de los primeros años de vida de estas sociedades no es fácil por la falta de documentación. Sin embargo, para el último cuarto del siglo la información es algo más completa gracias a la publicación, desde 1971 y bajo diversas denominaciones, del Anuario Estadístico de las Cooperativas de Crédito y Cajas Rurales y a la inclusión por parte del Banco de España del desglose provincial de actividad de estas entidades en su Boletín Estadístico. Tomando como base estas fuentes, además de otras informaciones, se realiza un estudio más detenido de la actividad de las cajas rurales andaluzas entre 1975 y 2000. Se diferencian dos áreas en la región (oriental y occidental) tratando de identificar rasgos distintivos en ambas.

Palabras clave: cajas rurales; sindicatos agrícolas; cooperativas agrícolas; entidades de crédito; expansión.

Claves Econlit: G21; N24; P13; Q14.

\section{[en] Andalusian credit cooperatives in the twentieth century: origin and consolidation of the sector}

Abstract. The main objective of this work is to analyze the history of Andalusian rural credit banks
during the twentieth century. We try to study the development from its origin to the consolidation of
the sector in the nineties. The first entities of this kind arise in Andalusia in the early years of this
century, somewhat later than they did in other European countries and with less push than in other
areas of Spain. Since then, numerous small-scale entities appeared linked to Agricultural Unions or as
credit sections of agricultural cooperatives. But they did not acquire the status of credit institution.
Since mid-century, the Andalusian sector of rural banks begins to take shape with institutions that
remain operational until the end of the century. The study of the early life of these companies is not
easy due to the lack of documentation. However, since the 1970s, the information is more complete

1 Universidad de Jaén, España

Dirección de correo electrónico: mjvargas@ujaen.es 
thanks to the publication of the Statistical Yearbook of Credit Unions and Rural Credit Banks, since 1971. The Statistical Bulletin of the Bank of Spain also begins to include provincial information of the activity of these. Based on these sources, a further study of the activity of the Andalusian rural banks between 1975 and 2000 is performed. Two areas are differentiated in the region (East and West) trying to identify distinguishing characteristics in each zone.

Keywords: rural credit banks; agricultural unions; rural cooperatives; credit institutions; expansion.

Sumario. 1. Introducción. 2. Origen de las cooperativas de crédito y las cajas rurales en España y en Andalucía. 3. El primer tercio del siglo XX. 4. De la posguerra a la gran reforma de la década de los setenta. 5. El último cuarto de siglo: crisis, saneamiento y recuperación. 6. Análisis comparado de la actividad de las cajas rurales andaluzas (1975-2000). 7. El siglo XXI: crisis económica y concentración del sector. Unas pinceladas. 8. Conclusiones. 9. Referencias bibliográficas.

Cómo citar: Vargas-Machuca, M.F. (2017) Las cooperativas de crédito andaluzas en el siglo XX: origen y consolidación del sector. REVESCO. Revista de Estudios Cooperativos, No 124, pp. 175-214. DOI: 10.5209/REVE.54925.

\section{Introducción}

Las sociedades cooperativas de cualquier naturaleza surgen como una fórmula para cubrir una necesidad común de un grupo de personas. En el caso de las cooperativas de crédito, el origen viene determinado por el requerimiento colectivo de recursos financieros en momentos en los que el acceso al crédito en condiciones eficientes se hace difícil para sus socios.

Actualmente, las cooperativas de crédito españolas son un conjunto de instituciones, en su mayoría de pequeña dimensión, que, bajo la forma jurídica de sociedades cooperativas, tienen por objeto la cobertura de las necesidades financieras de sus socios, aunque también de terceros ${ }^{2}$. En su origen, surgieron vinculadas a distintos ámbitos de actuación, de ahí que se puedan distinguir tres tipos: cajas rurales, populares y profesionales. Sin embargo, en los tres casos, el negocio ha evolucionado hacia una menor especialización operativa y la diferencia entre ellos se ha difuminado (Vargas Vasserot, 2015).

Las primeras cooperativas de crédito españolas surgen a finales del siglo XIX, con algo de retraso respecto a otros países europeos. A partir de entonces, la evolución del marco legal fue configurando un sector que, a pesar de su expansión geográfica y funcional, ha mantenido una estrecha vinculación territorial.

Si bien, con el tiempo, este grupo de instituciones se ha ido consolidando en el sistema bancario español, han mantenido siempre una cuota de mercado relativamente menor que los bancos y las cajas de ahorros. No obstante, su intensa afinidad territorial les ha permitido alcanzar una importancia relativa considerable en algunas localidades o zonas geográficas, llegando, incluso, a superar al resto de entidades bancarias (Palomo Zurdo, Sanchis Palacio y Gutiérrez Fernández, 2011).

El objetivo de este artículo es analizar el devenir de las cooperativas de crédito andaluzas durante el siglo XX. Desde sus orígenes en los primeros compases de la

2 Melián Navarro, Palomo Zurdo, Sanchis Palacio y Soler Tormo (2006) presentan una interesante recopilación de los principales trabajos de investigación en materia de cooperativas de crédito y grupos cooperativos. 
centuria hasta su consolidación en el último cuarto del siglo, cuando la cuota de mercado que estas entidades alcanzan en esta región supera con creces la media nacional. Igualmente, se tratará de analizar si esta consolidación se lleva a cabo con la misma intensidad en las dos partes en las que se suele dividir tradicionalmente la región: oriental y occidental ${ }^{3}$. El trabajo se refiere básicamente a cajas rurales puesto que, a pesar de que hubo alguna experiencia de cooperativas de crédito de otra naturaleza, su vida y su éxito fueron bastante efímeros.

El estudio de la historia de estas entidades no resulta sencillo debido, fundamentalmente, a la falta de documentación (Titos Martínez, 2003). Por un lado, no existen investigaciones específicas dedicadas al devenir de las mismas, salvo en el caso de la Caja Rural de Granada (Titos Martínez, 2005) y de Almería (Galdeano Gómez, 1994), aunque este último tan sólo hace una breve mención a algunos acontecimientos clave de la historia de la entidad, centrándose en la actividad de la misma entre 1987 y 1991 . Por otro lado, la disponibilidad de datos relativos a sus operaciones, especialmente para los primeros años de vida, es también muy limitada. El capítulo que Titos Martínez (2003) dedica a las Cajas Rurales en el contexto de la historia del sistema financiero andaluz es, probablemente, el trabajo más completo. No obstante, precisamente por esa ausencia de información, se centra tan sólo en las cajas rurales que operaban en esta región a comienzos de la década de los ochenta del siglo XX, sin mencionar a algunas que existieron con anterioridad y a otras cooperativas de crédito no agrarias. Existen otros estudios ${ }^{4}$ que analizan la actividad de las cajas rurales andaluzas, pero solo para un periodo de tiempo concreto, sin hacer una descripción detallada de sus inicios ni de su evolución durante los primeros años de existencia.

Para profundizar en el origen y en la actividad de estas entidades durante sus etapas iniciales sería necesario recurrir a las memorias que anualmente publica cada una de las ellas. No obstante, esto no siempre es fácil, ya que muchas de estas cajas rurales no guardan todos los ejemplares de dichos documentos. Por tanto, para los primeros años de este análisis se utilizará la información recogida en la bibliografía publicada al respecto. Sin embargo, en el último cuarto del siglo XX la disponibilidad de datos es mucho mayor. Para ese periodo se tendrán en cuenta los datos presentados por la Unión Nacional de Cooperativas de Crédito en su anuario estadístico que, con distintas denominaciones, se publica desde 1971 (salvo para 1975-1977). Aunque sus primeras ediciones eran algo escasas en contenidos, comenzaron a ordenar y sistematizar la información relacionada con este grupo de entidades. Asimismo, se utilizarán las cifras sobre la distribución territorial de las oficinas, los depósitos y los créditos de estas entidades en Andalucía recogidas por el Banco de España en su Boletín Estadístico. Igualmente se incorporarán los datos recogidos por el Banco de España en su Registro Oficial de Entidades acerca de cada una de estas instituciones. Toda esta documentación se completará, en los casos en los que sea necesario, con las series elaboradas por Titos Martínez (2003),

\footnotetext{
La distribución de las provincias entre estas dos partes de Andalucía es la siguiente. Andalucía Oriental: Málaga, Granada, Almería y Jaén. Andalucía Occidental: Córdoba, Sevilla, Huelva y Cádiz.

4 Rodero Franganillo (1980), Sánchez Antonijuan (1985), Martín Mesa (1987), González Alcón (1995), Nieto González, Belmonte Ureña y Nieto González (1998) y Martín Zamora, (2000).
} 
tomando como referencia, además del Anuario de la UNACC, las propias memorias de las cajas rurales con sede en esta comunidad autónoma a las que este autor pudo tener acceso.

El artículo se divide en seis partes siguiendo la evolución cronológica de estas entidades. En primer lugar, se analiza el germen de las cooperativas de crédito y las cajas rurales en España y en Andalucía. Seguidamente, se estudia el desarrollo de las cooperativas de crédito en tierras andaluzas a través de tres etapas: el primer tercio del siglo, de la posguerra a la gran reforma del sistema financiero español de la década de los setenta y el último cuarto de siglo. A continuación, se dedica un apartado a la evolución de su actividad en este último periodo, diferenciando dos ámbitos territoriales en la región: la zona oriental y la occidental. Antes de finalizar, se incluye una breve referencia al proceso de concentración del sector en los primeros años del siglo XXI. Por último, se presentan las principales conclusiones del trabajo, las referencias bibliográficas consultadas y tres anexos en los que se recogen los datos detallados por entidades de los recursos de terceros, inversión crediticia y número de sucursales.

\section{Origen de las cooperativas de crédito y las cajas rurales en España y en Andalucía}

Las sociedades cooperativas, en general, surgen por el deseo de un grupo de personas de cubrir un requerimiento común. En el caso de las cooperativas de crédito, el nacimiento viene determinado, igualmente, por la necesidad colectiva de recursos financieros, cuando el acceso al crédito en condiciones eficientes en el mercado se hace difícil para sus socios (Belmonte Ureña, 2007).

Las primeras cooperativas de crédito surgen en Europa en la segunda mitad del siglo XIX ${ }^{5}$, en un periodo de importantes tensiones políticas y económicas, unidas a la crisis de la agricultura europea (Soler Tormo, 2002). La reorganización y modernización de la producción agrícola implicaba fuertes inversiones de capital lo que, para muchos campesinos, se traducía en la necesidad de recurrir al crédito. Sin embargo, el escaso desarrollo del sistema financiero y la concentración del negocio bancario en muy pocas manos, dificultaba el acceso a la financiación de estos agentes que tenían que soportar tipos de interés abusivos. En este contexto, las cooperativas de crédito presentaban una respuesta adecuada ya que podían ofrecer intereses inferiores a los exigidos por los bancos y el resto de prestamistas privados (Martínez Soto, 1994).

La lucha contra la usura y el mutualismo de inspiración cristiana, serían la base sobre la que se desarrollaron las primeras cooperativas de crédito en Alemania (Soler Tormo, 2002). Estas entidades estaban basadas en las ideas de Friedrich W. Raiffeisen quien defendía el cooperativismo de crédito como la mejor solución para liberarse de la usura y de la necesidad de financiarse a través de los banqueros (Nieto González et al., 1998). Este sistema de cooperación y otros similares (Durand en Franca, Cerruti en Italia, etc.) así como otras modalidades de naturaleza

5 Un análisis más detallado del cooperativismo de crédito en Europa puede encontrarse en Belmonte Ureña (2007), Luque Frías (2012), Palomo Zurdo (1995 y 2004) y Vargas Vasserot (2015). 
laica (Mutualismo oficial en Francia, Luzatti y Wollemborg en Italia y SchulzeDelitzsch y Haas en Alemania, etc.) se extendieron rápidamente por todo el continente Europeo (Martínez Soto, 2000).

En el caso de España, las primeras entidades de este tipo surgen en los últimos años del siglo XIX con algo de retraso respecto a los países de Europa occidental ${ }^{6}$.

No obstante, hay autores (Belmonte Ureña, 2007, Titos Martínez, 2003) que consideran que las cooperativas de crédito tuvieron en España su antecedente en los denominados pósitos, instituciones sin ánimo de lucro que, desde el siglo XV, se fundan en la mayor parte de los pueblos españoles. En ellos se guardaba el excedente de grano para poder prestarlo a campesinos con necesidades o para destinarlo al consumo en épocas de escasez (Carasa Soto, 1983). Estas organizaciones tenían como uno de sus objetivos la lucha contra la usura. Buscaban, en última instancia, evitar el excesivo endeudamiento de los labradores, especialmente en su modalidad más onerosa como era el adelanto a cuenta de la cosecha futura (Tello Aragay, 1994).

Después de una evolución irregular durante varios siglos (Belmonte Ureña, 2007), los pósitos terminan convirtiéndose, en el siglo XIX, en instituciones de microcrédito agrícola administradas, en su mayor parte, por los ayuntamientos (Martínez Soto, 2007).

En Andalucía, durante la segunda mitad del siglo XIX, el número de pósitos se situó en torno a los seiscientos. De hecho, en 1907, esta región contaba con 593 instituciones de este tipo, lo que suponía un 17 por 100 del total de estas organizaciones a nivel nacional. No obstante, si se tiene en cuenta el volumen de créditos concedidos, en 1906 los andaluces representaban un 68 por 100 de los préstamos en grano y un 69 por 100 de los préstamos en dinero del total concedido por los pósitos españoles (Martínez Soto, 2007). Es decir, aunque los pósitos andaluces no fueron especialmente relevantes a nivel nacional en cuanto a su número, sí lo fueron en cuanto a su volumen de operaciones, manteniendo una actividad muy por encima del resto de organismos de este tipo (Bernal, 1991).

Posteriormente, la corrupción y los abusos, supusieron, en no pocos casos, la decadencia de estas instituciones que, en sus últimos años, carecían de gran provecho debido a sus escasos recursos y a la limitación del crédito que podían conceder (Rodero Franganillo, 1974). En el siglo XX, diversas reformas legislativas promovieron la posibilidad de transformar estas entidades en bancos agrícolas, cajas de ahorros y montes de piedad (Belmonte Ureña, 2007). En Andalucía, no obstante, en los primeros años del siglo XX, los pósitos siguieron teniendo cierta importancia aunque sus fondos no fueron suficientes para cubrir los ingentes recursos que requería la modernización del sector (Sánchez Picón y Navarro Pérez, 2001).

Si bien hubo algunos intentos previos de constitución de cooperativas de crédito como tales en España ${ }^{7}$, se puede decir que la primera aplicación práctica de las

6 Los orígenes de las cajas rurales españolas se analizan en profundidad en Noguer (1980) y la evolución legal e institucional posterior hasta 1982 en Terrón Muñoz (1987). En Palomo Zurdo y Valor Martínez (2001) se recoge, igualmente, un repaso a la historia del sector de las cooperativas de crédito en España así como un análisis de la organización y agrupación institucional de las mismas a lo largo del siglo XX.

7 En 1858 se crea en Valencia la "Cooperativa" de los Papeleros de Buñol que, sin tener jurídicamente naturaleza de cooperativa de crédito, en la práctica operaba como tal (Palomo Zurdo, 2004). Alrededor de 
ideas de Raiffeisen se concretó en 1881 mediante la constitución de le Caja Rural de Ahorros, Préstamos y Socorros de Jabalí Viejo en Murcia (Martínez Soto, 2003a). Desde entonces, el movimiento del cooperativismo de crédito experimentó un relativo impulso promovido, en muchos casos, por el clero católico y siguiendo el mismo modelo germano cuyos planteamientos habían traído a nuestro país algunos estudiosos del tema como Díaz de Rábago, el Padre Nogués y el Vizconde de Eza (Rodero Franganillo, 1974).

En los comienzos de siglo XX, la Administración, la Iglesia y determinados grupos de propietarios, impulsaron con interés la organización cooperativa en el ámbito rural (Garrido Herrero, 1995). De hecho, las primeras cooperativas de crédito que surgen en España en los albores del nuevo siglo lo hacen vinculadas casi exclusivamente a la actividad agraria (Conde Rodríguez, 1995). En 1901 se constituye la primera caja rural española en la localidad palentina de Amusco a la que le siguen otras en la provincia de Zamora y en la de Castellón. En 1904 se crea otra en la localidad navarra de Olite (Palomo Zurdo y Valor Martínez, 2001).

En Andalucía las primeras referencias acerca de las cooperativas de crédito se sitúan en 1902. Ese año fue nombrado Delegado de Hacienda en Granada Don Francisco Rivas Moreno, quien, movido por su preocupación por las clases más necesitadas, había fundado en años anteriores diversas instituciones de inspiración social como cajas de ahorros y cajas rurales en otras zonas de España ${ }^{8}$. En su nuevo destino, comienza las gestiones para poner en marcha organizaciones agrarias de este tipo en varias localidades. Aunque en la capital el planteamiento tuvo una mayor aceptación inicial, el traslado de Rivas Moreno a Valencia, tan solo seis meses después de su llegada, provocó el debilitamiento del entusiasmo y el fracaso del proyecto (Titos Martínez, 2005).

Todas esas primeras experiencias fueron las pioneras de un sistema que se vería impulsado con la aprobación, en 1906, de la Ley de Sindicatos Agrarios, que promovió la creación de numerosas cooperativas bajo la fórmula de "sindicatos agrícolas" y de cajas rurales, autónomas o ligadas a los anteriores (Martínez Rodríguez y Martínez Soto (2008).

Desde esa fecha, comienzan a extenderse con rapidez los Sindicatos Católicos Agrarios, muchos de ellos con caja rural, que se integraban en la Confederación Nacional Católica.

\section{El primer tercio del siglo $\mathrm{XX}$}

En 1909, el primer censo de sindicatos agrícolas y cajas rurales recoge un total de 129 sindicatos con caja rural en España, de los cuales, ocho tenían sede en Andalucía (Titos Martínez, 2003). A partir de ese año, la evolución de estas instituciones en este territorio fue algo irregular (Cuadro 1). El número de cajas rurales andaluzas se reduce en la década de los veinte en mayor proporción que lo

1865, aparece otra entidad en Madrid denominada Manantial de Créditos, que sí puede ser considerada como la primera cooperativa de crédito española (Palomo Zurdo y Valor Martínez, 2001).

8 Las ideas de Rivas Moreno sobre cooperativismo del crédito agrario y sus proyectos se explican con detalle en sus propias obras (Rivas Moreno, 1895 y 1902) y en la obra de López Yepes (2003). 
hace a nivel nacional y se recupera en los años previos a la Guerra Civil. Paralelamente, la importancia que esta región representaba en el conjunto nacional de estas entidades cae hasta 1918, aunque remonta ligeramente en los años siguientes.

Tabla. 1. Sindicatos con caja rural y Cajas Rurales en Andalucía (1909-1933).

\begin{tabular}{|c|c|c|c|c|c|}
\hline \multirow[b]{2}{*}{ Año } & \multicolumn{2}{|c|}{$\begin{array}{l}\text { Sindicatos con caja } \\
\text { rural }^{\text {a }}\end{array}$} & \multicolumn{2}{|c|}{ Cajas Rurales ${ }^{\mathrm{b}}$} & \multirow{2}{*}{$\begin{array}{c}\% \\
\text { And./Esp. }\end{array}$} \\
\hline & Andalucía & España & Andalucía & España & \\
\hline 1909 & 8 & 129 & - & - & 6,20 \\
\hline 1911 & 111 & 1.771 & - & - & 6,27 \\
\hline 1915 & - & - & 24 & 525 & 4,57 \\
\hline 1918 & - & - & 11 & 503 & 2,19 \\
\hline 1924 & - & - & 11 & 501 & 2,20 \\
\hline 1933 & - & - & 29 & 646 & 4,49 \\
\hline
\end{tabular}

a Según Censo de Sindicatos y Cajas Rurales

b Según Anuario Estadístico de España y Dirección General de Agricultura

Fuente: Elaboración propia con datos de Noguer (1980), pp. 552-553; Titos Martínez (2003), p. 268 y Martínez Soto (2003b), p. 132.

En términos globales, estas entidades y, en especial, las cajas rurales, experimentaron una fase de expansión en el primer tercio del siglo. No obstante, el proceso de crecimiento se detuvo con la Guerra Civil y se ralentizó los años posteriores (Garrido Herrero, 1995; Gámez Amián, 1998). Sin embargo, durante este periodo, el desarrollo de estas entidades en Andalucía fue menor al de otras zonas del país en las que el cooperativismo tenía una mayor relevancia, como puede ser el caso de Valencia, Cataluña o Castilla León (Rodero Franganillo, 1980).

Desde el punto de vista jurídico, la primera norma que regula, aunque brevemente, las cooperativas de crédito es el Real Decreto de 12 de julio de 1917 de fomento del crédito agrícola, que pretendía estructurar el sector, reuniendo a los organismos que, en ese momento, canalizaban los préstamos agrarios: pósitos, asociaciones agrarias, sindicatos agrícolas y cajas rurales. Reglamentaba las asociaciones cooperativas de crédito, como aquellas cuyo objeto social debería ser el crédito a los socios o a otras asociaciones cooperativas agrícolas (Terrón Muñoz, 1987).

En 1925, el Directorio Militar de la Dictadura crea el Servicio Nacional de Crédito Agrícola (Gámez Amián, 1998), organismo oficial que se encargaría de esta modalidad de financiación y que, en 1962, pasaría a denominarse Banco de Crédito Agrícola. 


\section{De la posguerra a la gran reforma de la década de los setenta}

Tras el paréntesis de la Guerra Civil, en 1942, se aprueba la Ley de Cooperación, con la que las cooperativas quedan vinculadas a la Organización Sindical y a la Unión Nacional de Uniones Territoriales de Cooperativas del Campo (Palomo Zurdo, 2004). Igualmente, esta Ley establecía una clasificación dentro del grupo de las cajas rurales: las secciones de crédito de las cooperativas del campo ${ }^{9}$ (dependientes de éstas), las cajas rurales de primer grado (locales y comarcales) y las de segundo grado o provinciales que tenían como socios a las cooperativas del campo de la provincia en la que establecieran su sede (Hidalgo Fernández y Bernabéu Aguilera, 2014; Server Izquierdo y Melián Navarro, 2001; Terrón Muñoz, 1987).

Una vez superadas las consecuencias más graves de la contienda, el número de cooperativas de crédito volvió a aumentar debido, no sólo a la urgencia de cubrir las nuevas necesidades financieras del desarrollo económico, sino también porque muchas fueron utilizadas por otras entidades para evitar las prohibiciones que imponía el statu quo en el que se vio inmerso el sistema bancario español durante los primeros veinte años del franquismo. Estas restricciones no afectaron al conjunto de las cooperativas de crédito que, por entonces, no se consideraban plenamente integradas en ese sector.

A partir de entonces, comienzan a extenderse por tierras andaluzas nuevas cajas rurales, fundamentalmente de carácter local, concentrándose de forma especial en las provincias de Córdoba, Granada, Jaén y Sevilla (Rodero Franganillo, 1974). Sin embargo, muchas de estas entidades no perdurarían en el tiempo ni llegarían a convertirse en verdaderas entidades financieras. De hecho, Terrón Muñoz (1987), con datos de la Caja Rural Nacional, recoge algunas instituciones en diversas localidades de Córdoba y Sevilla que se inscriben en el Ministerio de Trabajo pero que no llegan a hacerlo posteriormente en el Registro Oficial del Banco de España (Cuadro 2).

9 Las secciones de crédito que se forman en el interior de cooperativas de cualquier tipo tienen como finalidad de satisfacer las necesidades financieras de los socios y de otras secciones de la propia cooperativa. Si bien, es muy probable que los datos correspondientes a esos años de mediados de siglo contabilicen estas secciones como cajas rurales, no serán objeto de este trabajo puesto que, al carecer de personalidad propia e independiente, no se consideran parte integrante del sistema bancario español. De hecho, en la década de los setenta no se podrán registrar como cajas rurales en el Registro Oficial de Entidades del Banco de España. 
Tabla. 2. Cajas comarcales y locales andaluzas registradas en el Ministerio de Trabajo que no llegan a inscribirse en el Registro del Banco de España.

\begin{tabular}{|c|c|c|c|}
\hline Entidad & Población & $\begin{array}{l}\text { Año de } \\
\text { constitución }\end{array}$ & $\begin{array}{c}\text { Núm. } \\
\text { Registro } \\
\mathrm{M}^{\mathrm{o}} \\
\text { Trabajo }\end{array}$ \\
\hline $\begin{array}{l}\text { C.R. Cooperativa Olivarera Jesús } \\
\text { Nazareno }\end{array}$ & Bujalance (Córdoba) & 1947 & 3.323 \\
\hline C.R. Nuestra Señora de la Sierra & Cabra (Córdoba) & 1955 & 6.692 \\
\hline C.R. Nuestro Padre Jesús & La Rambla (Córdoba) & 1962 & 10.134 \\
\hline C.R. San Isidro Labrador & Marchena (Sevilla) & 1963 & 10.932 \\
\hline $\begin{array}{l}\text { C.R. Filial Cooperativa Olivarera } \\
\text { Pontanense }\end{array}$ & Puente Genil (Córdoba) & 1956 & 6.907 \\
\hline C.R. Nuestra Señora de la Estrella & Villa del Río (Córdoba) & - & 5.942 \\
\hline
\end{tabular}

$$
\text { C.R. = Caja Rural }
$$

Fuente: Elaboración propia con datos de Terrón Muñoz (1987, pp. 425).

Igualmente, en el Registro de Sociedades Cooperativas de la Junta de Andalucía, además de estas, aparecen registradas algo más de treinta cooperativas de crédito constituidas entre 1947 y 1965 de las que después tampoco existe constancia en el Registro de Entidades del Banco de España ${ }^{10}$. En la mayoría de los casos se trataría de cajas rurales locales o secciones de crédito de cooperativas agrícolas que, por aquel entonces, también se denominaban cajas rurales, y que luego no llegan a cumplir los requisitos necesarios para poder adquirir la condición de entidad de crédito bajo la supervisión del Banco de España.

A pesar de todas estas experiencias que no llegan a consolidarse, se puede decir que, a partir de finales de los cincuenta se inicia la configuración "moderna" del sistema de cajas rurales de la región. Se trata de entidades que, una vez registradas en el Banco de España, se mantienen, en su mayoría hasta acabar el siglo XX. En el Cuadro 3 se recoge la cronología de constitución de estas cooperativas y su evolución posterior.

En 1957 se constituye la Caja Rural Nacional (CRUNA) como sociedad cooperativa de crédito de segundo grado, con la intención de resolver las dificultades que empezaban a vislumbrar debido a la falta de unión entre estas entidades de crédito cooperativo rural en España (Belmonte Ureña, 2007). Esta institución contribuyó al despegue definitivo del sector en los comienzos de la

10 De estas entidades solo aparecen en dicho Registro la denominación (en algunos casos incompleta), el número de inscripción y la fecha de constitución. Ante la falta de información más detallada se ha optado por no recoger esta relación a la que puede accederse a través de la Consulta Pública de Registro de Cooperativas de la Junta de Andalucía:

(http://www.juntadeandalucia.es/economiainnovacionyciencia/ConsultaSociedades/rscl/inicioConsultaSocieda des.do). 
década de los sesenta y, en especial, a la constitución de cajas rurales provinciales (Palomo Zurdo y Valor Martínez, 2001). Fruto de este impulso, ese mismo año nace la primera de las cooperativas de crédito "modernas" de Andalucía: la Caja Rural Provincial de Jaén, entidad que mantiene su actividad hasta nuestros días.

En los siguientes trece años se fundan en Andalucía un total de 16 cajas rurales y 4 cooperativas de crédito no agrarias. Cabe destacar la primacía de la provincia de Córdoba como destino de estas nuevas sociedades ya que en ese territorio se instalan 7 de ellas.

La evolución de estas entidades es muy heterogénea. Del grupo de las cajas rurales provinciales, todas mantienen su actividad hasta finales de siglo ${ }^{11}$, salvo la de Cádiz que sería adquirida por la Caja de Ahorros de San Fernando en 1986. Años después, en la transición de siglo, las de Málaga, Almería, Huelva y Sevilla se verán inmersas en sendos procesos de concentración de los que surgirán Cajamar y la Caja Rural del Sur, con sedes en Almería y Sevilla respectivamente.

Tabla. 3. Cooperativas de crédito fundadas en Andalucía desde 1957 y evolución posterior (Entidades que llegan a registrarse en el Banco de España).

\begin{tabular}{|c|c|c|c|c|c|}
\hline \multirow[b]{2}{*}{ Entidad } & \multirow[b]{2}{*}{ Ámbito } & \multirow[b]{2}{*}{ Provincia } & \multirow{2}{*}{$\begin{array}{c}\text { Año } \\
\text { fundación } \\
\end{array}$} & \multicolumn{2}{|r|}{ Desaparición } \\
\hline & & & & Año & Causa \\
\hline \multicolumn{6}{|l|}{ CAJAS RURALES } \\
\hline C.R. Provincial de Jaén & $\mathrm{P}$ & Jaén & 1957 & - & - \\
\hline $\begin{array}{l}\text { Soc. Coop. C.R. San José } \\
\text { (Torredonjimeno) }\end{array}$ & $\mathrm{L}$ & Jaén & 1958 & 1989 & $\begin{array}{l}\text { Transformación en sección de } \\
\text { crédito de coop. Agraria }\end{array}$ \\
\hline $\begin{array}{l}\text { C.R. Coop. de Crédito } \mathrm{N}^{\mathrm{a}} \mathrm{S}^{\mathrm{a}} \text { del } \\
\text { Rosario (Nueva Carteya) }\end{array}$ & $\mathrm{L}$ & Córdoba & 1958 & - & - \\
\hline $\begin{array}{l}\text { Coop. de Crédito C. R. } \mathrm{N}^{\mathrm{a}} \mathrm{S}^{\mathrm{a}} \text { Madre } \\
\text { del Sol (Adamuz) }\end{array}$ & $\mathrm{L}$ & Córdoba & 1959 & - & - \\
\hline $\begin{array}{l}\text { Coop. de Crédito C. R. Los } \\
\text { Pedroches (Pozoblanco) }\end{array}$ & $\mathrm{L}$ & Córdoba & 1961 & 1979 & $\begin{array}{l}\text { Absorción por Caja Rural } \\
\text { Provincial de Córdoba }\end{array}$ \\
\hline $\begin{array}{l}\text { C.R. Coop. de Crédito } \mathrm{N}^{\mathrm{a}} \mathrm{S}^{\mathrm{a}} \text { del } \\
\text { Campo (Cañete de las Torres) }\end{array}$ & $\mathrm{L}$ & Córdoba & 1962 & - & - \\
\hline $\begin{array}{l}\text { Coop. de Crédito C. R. } \mathrm{N}^{\mathrm{a}} \mathrm{S}^{\mathrm{a}} \text { de } \\
\text { Guadalupe (Baena) }\end{array}$ & $\mathrm{C}$ & Córdoba & 1962 & - & - \\
\hline C.R. Provincial de Málaga & $\mathrm{P}$ & Málaga & 1962 & 2000 & $\begin{array}{l}\text { Fusión con Caja Rural de Almería } \\
\text { en Cajamar }\end{array}$ \\
\hline C.R. Provincial de Almería & $\mathrm{P}$ & Almería & 1963 & 2000 & $\begin{array}{l}\text { Fusión con Caja Rural de Málaga } \\
\text { en Cajamar }\end{array}$ \\
\hline C.R. Provincial de Córdoba & $\mathrm{P}$ & Córdoba & 1964 & 2014 & $\begin{array}{l}\text { Fusión por absorción con Caja } \\
\text { Rural del Sur }\end{array}$ \\
\hline C.R. Provincial de Sevilla & P & Sevilla & 1964 & 2001 & $\begin{array}{l}\text { Fusión con Caja Rural de Huelva } \\
\text { en Caja Rural del Sur }\end{array}$ \\
\hline
\end{tabular}

11 Durante la década de los noventa, todas estas cajas dejan de tener el calificativo de provinciales. 


\begin{tabular}{|c|c|c|c|c|c|}
\hline C.R. Utrera & $\mathrm{L}$ & Sevilla & 1964 & - & - \\
\hline $\begin{array}{l}\text { Coop. de Crédito C. R. Caja Rural } \\
\text { de La Carlota }\end{array}$ & $\mathrm{L}$ & Córdoba & 1965 & 1991 & $\begin{array}{l}\text { Absorción por Caja Rural } \\
\text { Provincial de Córdoba }\end{array}$ \\
\hline C.R. Provincial de Huelva & $\mathrm{P}$ & Huelva & 1967 & 2001 & $\begin{array}{l}\text { Fusión con Caja Rural de Sevilla en } \\
\text { Caja Rural del Sur }\end{array}$ \\
\hline $\begin{array}{l}\text { Coop. de Crédito C.R. de Morón de } \\
\text { la Frontera }\end{array}$ & $\mathrm{L}$ & Sevilla & 1968 & 1980 & $\begin{array}{l}\text { Baja por acuerdo del Consejo } \\
\text { Ejecutivo }^{(1)}\end{array}$ \\
\hline C.R. Provincial de Cádiz & $\mathrm{P}$ & Cádiz & 1969 & 1986 & $\begin{array}{l}\text { Adquisición por Caja de Ahorros } \\
\text { de San Fernando }\end{array}$ \\
\hline C.R. Provincial de Granada & $\mathrm{P}$ & Granada & 1970 & - & - \\
\hline $\begin{array}{l}\text { Caja Rural de Almería y Málaga } \\
\text { (Cajamar) }\end{array}$ & - & Almería & 2000 & - & - \\
\hline $\begin{array}{l}\text { Caja Rural del Sur (Huelva y } \\
\text { Sevilla) }\end{array}$ & - & Sevilla & 2001 & - & - \\
\hline
\end{tabular}

\begin{tabular}{|c|c|c|c|c|c|}
\hline $\begin{array}{l}\text { OTRAS COOPERATIVAS DE } \\
\text { CRÉDITO }\end{array}$ & & & & & \\
\hline $\begin{array}{l}\text { Central del Ahorro Popular, Coop. } \\
\text { de Crédito }\end{array}$ & - & Sevilla & 1963 & 1974 & $\begin{array}{l}\text { Absorción por Central del Ahorro } \\
\text { Popular Madrid }\end{array}$ \\
\hline Caja Latina de Crédito, Soc. Coop. & - & Córdoba & 1966 & 1975 & Disolución \\
\hline $\begin{array}{l}\text { Cooperativa de Crédito y Fomento } \\
\text { Industrial }\end{array}$ & - & Córdoba & 1967 & 1976 & $\begin{array}{l}\text { Baja por acuerdo de la C. Ejecutiva } \\
\text { del Banco de España }\end{array}$ \\
\hline Caja de Crédito del Sur & - & Málaga & 1969 & 1979 & Declaración de quiebra voluntaria \\
\hline
\end{tabular}

\section{C.R. = Caja Rural}

Ámbito = P (Provincial), C (Comarcal) y L (local)

${ }^{(1)}$ Baja en el Registro Oficial de Entidades del Banco de España.

Fuente: Elaboración propia con datos del Anuario Estadístico de las Cooperativas de Crédito (1973 y 1974), Titos Martínez (2003, pp. 269-270) y Registro Oficial de Entidades del Banco de España

Entre las de dimensión local o comarcal, se producen más cambios. La Caja Rural Provincial de Córdoba absorbe a la de Los Pedroches en 1979 y a la de La Carlota en 1991. Por otro lado, la Caja Rural San José de Torredonjimeno se disuelve en 1989, transformándose en la sección de crédito de una cooperativa aceitera del mismo nombre, toda vez que el Banco de España no autorizara su absorción por parte de la Caja Rural Provincial de Jaén. Respecto a la Caja Rural de Morón de la Frontera (Sevilla), en el Registro de Entidades del Banco de España hay constancia de que causa baja en febrero de 1980 por acuerdo del Consejo Ejecutivo pero no hay información más concreta sobre las causas que la motivaron ni el destino específico de la entidad.

La experiencia de las cooperativas de crédito no agrarias fue bastante más limitada. De hecho, desparecen muy pronto del panorama bancario regional. La Caja Central del Ahorro Popular de Sevilla (1963-1974) es absorbida por la misma entidad de Madrid. Un año después se disuelve la Caja Latina de Crédito de 
Córdoba (1966-1975). En 1976 causa baja en el Registro de Entidades del Banco de España la cordobesa Cooperativa de Crédito y Fomento Industrial (1967-1976) y pasados tres años, se declara en quiebra la Caja de Crédito del Sur (1969-1979), que tenía su sede en Málaga. El hecho de que esta modalidad de cooperativas de crédito tuviera tan poco éxito en esta región puede llevar a pensar que, en muchos casos, las cajas rurales andaluzas han servido para cubrir las necesidades financieras de cooperativas no agrarias que, en otras zonas, han sido atendidas por cooperativas de crédito populares o profesionales (Carbonel de Masy, 1973).

Además de las entidades con sede social en Andalucía que recoge el Cuadro 3, al finalizar 1973 operaban en esta región tres cooperativas de crédito foráneas todas ellas de carácter no agrario y procedentes de Madrid: Crédito y Previsión, con oficinas en Cádiz, Córdoba y Granada; Caja Ibérica de Crédito, con presencia en Málaga, y La Agrupación de Cooperativas Farmacéuticas Acofar, con establecimiento en Cádiz, Jaén y Sevilla. Las dos primeras causarían baja en 1975 al ser absorbidas por sendos bancos nacionales, mientras que la última sería el germen del actual Bancofar, entidad que, a día de hoy, aún sigue manteniendo sucursales en territorio andaluz (UNACC, 1973; Banco de España, Registro Oficial de Entidades).

Por tanto, durante las décadas de los sesenta y setenta el sector se enfrenta a un doble proceso. Por un lado, surgen nuevas entidades, tanto de ámbito provincial como de ámbitos inferiores por la conversión de muchas secciones de crédito de cooperativas agrarias en cajas rurales. Por otro, tiene lugar una importante reestructuración que afecta de forma significativa a estas últimas, de forma que desaparecen, quedan integradas en otras más grandes a modo de nuevas sucursales o son absorbidas por parte de las provinciales (Martín Mesa, 1988). Como consecuencia el número global de estas entidades se ve reducido.

Se trata de un proceso a nivel nacional. De hecho, entre 1965 y 1973 el número de cajas rurales en España se contrae drásticamente de 3.587 a 207 (Cuadro 4). El caso andaluz, para el cual no se disponen de datos concretos para 1965, debió presentar una evolución en la misma línea. De hecho, si tenemos en cuenta que en 1933 se tenía constancia de la existencia 29 cajas rurales andaluzas y que en el Registro de Cooperativas de la Junta de Andalucía figuran más de 30 cooperativas de crédito constituidas hasta el año 1965 (aunque no todas rurales), el hecho de que en 1973 sólo se mantengan 21, puede llevar a afirmar que, de algún modo, también esta región experimentó el proceso de reestructuración desarrollado a nivel nacional. 
Tabla. 1. Número de cajas rurales y cooperativas de crédito en España y Andalucía (19651974)

\begin{tabular}{rrrrr}
\hline & \multicolumn{2}{c}{ España } & & Andalucía \\
\cline { 2 - 3 } Año & $\begin{array}{c}\text { Cajas } \\
\text { Rurales }\end{array}$ & $\begin{array}{c}\text { Total Coop. } \\
\text { de Crédito }\end{array}$ & & $\begin{array}{c}\text { Total Coop. } \\
\text { de Crédito }\end{array}$ \\
\hline 1965 & 3.587 & - & - \\
1966 & 1.098 & - & - \\
1967 & 589 & - & - \\
1968 & 618 & - & - \\
1969 & - & 730 & & - \\
1973 & ${ }^{a} 207$ & 242 & & 21 \\
1974 & ${ }^{b} 226$ & 262 & & 21
\end{tabular}

a 124 de ellas en trámite de autorización por parte del Banco de España.

b 115 de ellas en trámite de autorización por parte del Banco de España.

Fuente: Elaboración propia con datos de Titos Martínez (2003), p. 268; Palomo Zurdo y Valor Martínez (2001), p. 43-45, Anuario Estadístico de las Cooperativas de Crédito (1973 y 1974); Anuario Cajas Rurales, Populares y Profesionales (1995 y 2000) y Boletín Estadístico del Banco de España.

En paralelo a la configuración del sector ${ }^{12}$, desde el punto de vista legislativo, a mediados de la década de 1960 se inicia a nivel nacional un proceso de reformas legales que suponen el fin del statu quo bancario, que se había mantenido durante los años anteriores con la relajación de las barreras de entrada al sector y de apertura de sucursales. Además, estas medidas implicarían el inicio del proceso de la incorporación de las cooperativas de crédito al sistema financiero español ${ }^{13}$.

En 1964, el Decreto de 26 de marzo, supuso la reorganización de las funciones de la Caja Rural Nacional y el sometimiento de las cajas rurales a la cobertura de coeficientes y al control del Ministerio de Hacienda. Asimismo, reconocía numerosos beneficios para aquellas cajas que obtuvieran del Banco de España la categoría de "calificadas". Entre otros, se les permitía participar en la distribución del crédito oficial del Banco de Crédito Agrícola en similares condiciones a las que tenían el resto de entidades colaboradoras (Terrón Muñoz, 1987). Esto estimuló la creación de cajas rurales provinciales, promovidas muchas veces por las cajas locales ya existentes, con la intención de poder acceder al crédito agrario oficial y reorganizar sus formas de federación y consolidación. De hecho, Andalucía llega a contar con ocho de estas entidades, una en cada provincia, todas ellas "calificadas"

12 En el trabajo de Palomo Zurdo y Valor Martínez (2001, pp. 48-49) se recoge un cronograma del sector de las cooperativas de crédito en España que incluye sus antecedentes y los hechos más relevantes de su evolución histórica hasta la creación de la Asociación Española de Cajas Rurales en 1989.

13 Un análisis de estas reformas puede verse en Pons (2011). 
excepto la de Cádiz $^{14}$. A la vez, se extiende también la creación de Uniones Territoriales de Cooperativas (UTECOs) estrechamente vinculadas a las cajas rurales o a otras cooperativas de crédito (Palomo Zurdo y Mateu Gordon, 2013).

Finalmente, en 1971, se aprueba la autonomía de estas instituciones que quedan sometidas, de forma exclusiva, a la autoridad del Banco de España. Sin embargo, la norma más relevante de esta fase de reformas sería el Real Decreto 2860/78 de 3 de noviembre sobre cooperativas de crédito. Esta disposición y su desarrollo mediante diversas órdenes ministeriales de los dos años siguientes supusieron la integración total y definitiva de las cooperativas de crédito en sistema financiero español.

\section{El último cuarto de siglo: crisis, saneamiento y recuperación}

La favorable evolución de la actividad de estas entidades se ve interrumpida por la importante crisis que, entre 1971 y 1983, en un entorno de dificultad bancaria generalizada, alcanzó a un gran número de cajas rurales ${ }^{15}$. Esta situación, que afectó a veinte cajas rurales en todo el país, tuvo graves consecuencias en cuatro cajas andaluzas, en especial en la Caja Rural Provincial de Jaén. Esta entidad fue intervenida en 1982 por el Banco de España y necesitó un volumen muy considerable de ayudas financieras públicas (27.160 millones de pesetas) para hacer frente a la situación planteada por su excesiva concentración de riesgos con UTECO y con la Cooperativa Provincial Agrícola de Jaén (Martin Mesa, 1986). Igualmente, la Caja Rural Provincial de Sevilla fue intervenida por el Banco de España en 1983, aunque consiguió resolver la situación. La Caja de Málaga también necesitó un importante plan de saneamiento en 1984 con el que fue capaz de recuperarse, mientras que la de Cádiz terminó siendo adquirida por la Caja de Ahorros de San Fernando en 1986 (Titos Martínez, 2003).

Esta recesión implicó el establecimiento de un Plan de Saneamiento (entre 1983 y 1992) y la firma de un convenio de asociación de las Cajas Rurales y el Banco de Crédito Agrícola con objeto de dar mayor solidez al sector y proporcionarle un respaldo que garantizara su futuro ${ }^{16}$.

En mayo de 1984 se constituye el Grupo Asociado BCA-CRA ${ }^{17}$ entre el Banco de Crédito Agrícola y 57 Cajas Rurales, a las que se unieron posteriormente algunas más. Todas las cajas rurales andaluzas, a excepción de la de Torredonjimeno y la Caja Rural Provincial de Cádiz, se integran desde el principio en este proyecto (Martín Mesa, 1987). Este convenio favoreció el saneamiento de las cajas implicadas en la crisis, su reorganización y la canalización, a través de ellas, del crédito agrario oficial, lo que supuso un nuevo impulso para el sector. Igualmente, este acuerdo implicó la disolución de la Caja Rural Nacional, que se había constituido en 1970, cuyas funciones fueron absorbidas por el Grupo.

\footnotetext{
14 Según datos del Anuario Estadístico de las Cooperativas de Crédito de 1973 y 1974.

15 Un análisis detallado de la crisis del sector de las cajas rurales puede encontrarse en Martín Mesa (1988).

16 El Banco de Crédito Agrícola estaba clasificado como entidad oficial de crédito siendo su único accionista el Instituto de Crédito Oficial (Palomo Zurdo, 1995).

17 Grupo Asociado Banco de Crédito Agrícola - Cajas Rurales Asociadas.
} 
Simultáneamente, en 1983, se crea el Fondo de Garantía de Depósitos de las Cooperativas de Crédito, que también desempeñará un papel relevante en el proceso de saneamiento del sector.

El convenio con el Banco de Crédito Agrícola comienza a debilitarse cuando, en 1989, algunas cajas rurales deciden abandonarlo y crear la Asociación Española de Cooperativas de Crédito, como entidad de cohesión del sector, y el Banco Cooperativo Español, como su rama operativa bancaria. En la constitución de esta nueva entidad participaron las 24 cajas promotoras del proyecto en colaboración con el banco cooperativo alemán Deutsche Genossenschaftsbank (DG Bank) (Palomo Zurdo, 1995). De las entidades andaluzas, se incorporan inicialmente a este proyecto las siete cajas provinciales que participaban en el Grupo Asociado BCA-CRA (todas salvo la gaditana). Las cinco cajas de ámbito local y comarcal que tenían actividad en esa fecha se mantienen en este último Grupo, aunque se incorporarían al Banco Cooperativo años después.

Junto con el recién creado banco y como instrumentos comunes de gestión, se crearon dos empresas con cometidos especializados: la sociedad Rural de Servicios Informáticos y el grupo de seguros Rural Grupo Asegurador (RGA). Estos cuatro organismos terminarán actuando corporativamente bajo la denominación "Grupo Caja Rural", especialmente a partir de $1995^{18}$.

La disolución del convenio también se vería favorecida por el proceso de reestructuración de la banca pública española de comienzos de la década de los noventa que llevaría, en 1991, a la integración del Banco de Crédito Agrícola en la Corporación Bancaria de España Argentaria que, a su vez, sería privatizada en los años siguientes (Palomo Zurdo y Valor Martínez, 2001).

Por tanto, al iniciarse la década de los noventa, en Andalucía existían dos grupos en el sector de las cooperativas de crédito: las que seguían vinculadas al convenio con el Banco de Crédito Agrícola y las que se habían incorporado al Banco Cooperativo Español. A nivel nacional, a estos dos grupos había que añadirle uno más formado por el conjunto de las cajas rurales de Castellón que se mantenían vinculadas a Credicoop. Sin embargo, progresivamente, la mayoría de ellas se fueron incorporando al segundo grupo ${ }^{19}$.

La Ley de Cooperativas de Crédito de 1989 y su Reglamento de 1993 suponen un nuevo modo de entender estos intermediarios financieros en su doble vertiente de sociedades cooperativas y entidades de crédito. Sin embargo, esta regulación posee un carácter supletorio respecto de la aprobada por las Comunidades Autónomas con competencias legislativas en esta materia. Así, en Andalucía, el Decreto 158 de 10 de agosto de 1983, reguló las competencias del gobierno autonómico en relación a las cooperativas de crédito. En 1985 se promulgó la Ley de Sociedades Cooperativas Andaluzas, siendo sustituida por un nuevo texto legal con la aprobación de la Ley 2/1999 de 31 de marzo. Esta nueva ley establece, entre otras cuestiones, un sistema de control e inspección que es asumido como competencia del gobierno autonómico.

18 La concentración de las cooperativas de crédito a través de la formación de grupos responde a una estrategia de integración que les permite seguir operando en el mercado financiero bajo unos principios comunes y solidarios pero sin perder la ventaja competitiva individual de cada una de ellas (Melián Navarro, 2004).

19 Las únicas cajas rurales que para el año 2000 no se habían integrado en el Grupo Caja Rural son la Caja Rural Vasca y las cajas rurales locales de Castelldans y Guissona en Lérida. (Palomo Zurdo, 2004). 
La profunda crisis que el conjunto de las cajas rurales experimentó en los primeros años de la década de los ochenta supuso, entre otras cosas, una importante disminución del número de estas entidades en España. Si en 1977 se registraban 200, en 1985 eran 146 y cinco años después esa cifra ascendía tan solo a 107 (Cuadro 5). Estas reducciones responden, en muchos casos, a absorciones, bien por parte de otras cajas rurales, o bien por bancos o cajas de ahorros, aunque también se produjeron algunas liquidaciones (Palomo Zurdo y Valor Martínez, 2001). En el caso de Andalucía también se produce esta reducción aunque con menor intensidad pasando de 18 a 13 entidades entre 1977 y 1990 (Cuadro 5).

Tabla. 2. Número de cooperativas de crédito en Andalucía y España (1977-2000)

\begin{tabular}{rrrr}
\hline Año & Andalucía & España & $\begin{array}{c}\text { And/Esp } \\
(\%)\end{array}$ \\
\hline 1977 & 18 & 200 & 9,0 \\
1981 & 15 & 147 & 10,2 \\
\hline 1982 & 15 & 154 & 9,7 \\
1983 & 15 & 153 & 9,8 \\
\hline 1984 & 15 & 149 & 10,1 \\
1985 & 15 & 146 & 10,3 \\
\hline 1986 & 14 & 138 & 10,1 \\
1987 & 14 & 129 & 10,9 \\
\hline 1988 & 14 & 117 & 12,0 \\
1989 & 13 & 110 & 11,8 \\
\hline 1990 & 13 & 107 & 12,1 \\
\hline 1995 & 12 & 95 & 12,6 \\
\hline 2000 & 11 & 92 & 12,0 \\
\hline
\end{tabular}

Fuente: Elaboración propia con datos de Titos Martínez (2003), p. 268, Anuario Estadístico Cajas Rurales y Cooperativas de Crédito (1980), Anuario de las Cooperativas de Crédito y Cajas Rurales (1985), Anuario Cajas Rurales, Populares y Profesionales (1995 y 2000) y Boletín Estadístico del Banco de España.

Una vez superadas las dificultades ${ }^{20}$, las entidades continúan su actividad hasta los últimos años del siglo en los que tiene lugar un nuevo impulso del proceso de concentración del sector, La primera experiencia en este sentido tiene lugar en Andalucía donde, en el año 2000, surge Cajamar Caja Rural como resultado de la fusión de la Caja Rural de Almería y la Caja Rural de Málaga, entidades que, a su vez, habían integrado con anterioridad a la cooperativa agrícola y caja rural

20 Véase en González Alcón (1995) la evolución de las cajas rurales andaluzas en la década posterior a la crisis del sector. 
murciana del Campo de Cartagena (1989) y a la cooperativa de crédito madrileña Grumeco (2000). Se trata del primer caso de constitución de un SIP ${ }^{21}$ (Sistema Institucional de Protección) en España (Palomo Zurdo y Gutiérrez Fernández, 2012). De este modo, al acabar el siglo XX, tras esta nueva fase de integración, el número de cooperativas de crédito con sede en Andalucía era de 11, todas ellas de carácter agrario.

Se trata de un proceso de concentración del sector a nivel nacional, como consecuencia del ajuste generalizado del sistema bancario español (Palomo Zurdo y Sanchis Palacio, 2010; Melián Navarro, 2004). Esta tendencia se intensificará en la primera década del siglo XXI motivada, no tanto por las decisiones de las autoridades, sino por la voluntad del propio sector (Fajardo García, 2011).

Por otro lado, durante los últimos años del siglo XX, a nivel nacional el sector inicia un proceso de expansión territorial que lleva a las cooperativas de crédito a un importante crecimiento en el número de sus oficinas. Un modelo de negocio de banca minorista basado en la proximidad al cliente unido a otros factores como la intensificación de la competencia en el sistema financiero, la necesidad de encontrar nuevas oportunidades de negocio y los cambios en su operativa tradicional, llevaron a etas entidades a salir de sus ámbitos habituales de actuación. Esta expansión comenzó generalmente en las provincias o comarcas limítrofes en las que no había entidades de este tipo. Pero, con el tiempo, se fue extendiendo a zonas dentro del radio de acción de otras cooperativas de crédito que se convertían en competidoras (Palomo Zurdo y Sanchis Palacio, 2008). En ocasiones, el crecimiento territorial se derivaba de los propios procesos de fusiones y adquisiciones de entidades con áreas geográficas de actuación distintas. Este proceso supuso un sobredimensionamiento de la red de sucursales de estas entidades (al igual que ocurrió con las cajas de ahorros) que luego supondría un verdadero lastre cuando la crisis financiera afectara de lleno al sistema bancario español en los primeros años del siglo XXI (Gutiérrez Fernández, Palomo Zurdo y Romero Cuadrado, 2012).

En este contexto expansivo, durante los últimos años del siglo XX la Caja Rural de Almería mantiene un ritmo de crecimiento de su red de distribución muy por encima de la media del resto de cajas rurales andaluzas y españolas. De hecho, cuando en el año 2000 surge Cajamar por su fusión con la Caja Rural de Málaga, las 567 oficinas de la nueva entidad representan un 49 por 100 del total de las sucursales de las cajas rurales andaluzas y un 15 por 100 del total de cooperativas de crédito a nivel nacional. El resto de entidades con sede en Andalucía mantienen una evolución más acorde con la media del país, a excepción de las cajas locales que, al acabar el siglo XX mantienen únicamente una oficina situada en su localidad de origen.

21 Se trata de una nueva forma de integración de empresas que había surgido con la Directiva 2006/48/CE Directiva, traspuesta al ordenamiento jurídico español en 2009 a través de diversas disposiciones. En el caso de las cooperativas de crédito suponía agrupar bajo una entidad que actuaba como cabecera del grupo a una serie de cooperativas más pequeñas que mutualizaban sus resultados y ponderaban sus riesgos internos al $0 \%$, a la vez que les permitía mantener su identidad y marca comercial a la vez que cierta independencia jurídica y operativa (Puede verse en Palomo Zurdo y Gutiérrez Fernández (2012) una explicación más detallada de esta fórmula jurídica). 
Como se ha señalado, esta expansión geográfica no se limita a la región. Una vez que se flexibilizan los criterios legales para ello, en estas entidades aumenta el interés por crecer fuera de sus tradicionales ámbitos de actuación, tanto geográficos como operativos (Soler Tormo, 2002). Esta tendencia llevó a la Caja Rural de Almería a comenzar a operar en la provincia de Murcia (1989) al integrar la red de la Caja del Campo de Cartagena, absorbida por la entidad ese año, y a adquirir la red de sucursales que el Banco Urquijo mantenía en el área metropolitana de Barcelona (1990). La Caja Rural de Málaga expande también su zona de actuación con oficinas en Cádiz (1990), Madrid (1992), Melilla (1996) y Ceuta (1998). De igual modo, la Caja Rural de Granada compra al Banco Zaragozano seis de sus oficinas en Galicia (2001) aunque terminaría vendiéndolas a la Caja Rural Gallega un año después (Titos Martínez, 2005).

Sin embargo, a pesar de esta expansión geográfica, las cajas rurales mantuvieron una importante vinculación territorial. De igual modo, la homogeneización operativa con otras entidades y la pérdida de especialización no impidieron que estas instituciones consiguieran mantener algunos rasgos diferenciadores de su actividad respecto de la del resto de componentes del sistema bancario. No obstante en los últimos años, las cajas rurales y, en general, todas las cooperativas de crédito, han dado la imagen de querer suavizar su componente estrictamente agrario o rural, para remarcar un nuevo estilo de banca regional destinada a familias y pequeñas y medianas empresas (Soler Tormo, 2002; Vargas Vasserot, 2015).

\section{Análisis comparado de la actividad de las cajas rurales andaluzas (1975- 2000)}

Teniendo en cuenta el análisis realizado anteriormente del devenir de las cooperativas de crédito andaluzas que llegaron a inscribirse en el Banco de España, se puede decir que, desde finales de la década de los setenta, las única entidades de este tipo que mantienen su sede en la región son de carácter agrícola, de ahí que, a partir de ahora, nuestro estudio se centre en ellas.

El principal rasgo a destacar entre las cajas rurales andaluzas es la heterogeneidad en el tamaño. De todas ellas destaca, de modo especial, la Caja Rural Provincial de Almería que, antes de finalizar el siglo, llega a convertirse en la primera caja rural a nivel nacional por tamaño (Titos Martínez, 2003). El resto de entidades desarrollan una actividad algo más reducida, especialmente en el caso de las de dimensión local o comarcal.

Para analizar la actividad desarrollada por las cajas rurales en Andalucía hasta el año 2000 se estudiarán, de forma diferenciada y comparada, las dos partes en las que podemos dividir la región: oriental y occidental ${ }^{22}$. Se tendrá en cuenta la evolución en ambas zonas del número de entidades, volumen de recursos ajenos e inversión crediticia, sucursales y cuotas de mercado.

En lo que se refiere al número de entidades con sede en cada una de las dos áreas, es manifiesta la primacía de la parte occidental que, en estos años, llega a

22 Véase Nota al pie 2. 
contar con diez ${ }^{23}$ cajas rurales mientras que en la oriental mantiene su sede tan solo $\operatorname{cinco}^{24}$.

Sin embargo, desde el punto de vista de los recursos de terceros (Anexos 1 y 2) destaca la prioridad de la zona oriental, a pesar del menor número de entidades con sede en ella. Esto es debido, básicamente, a la superioridad de la Caja Rural de Almería desde mediados de los años ochenta. De hecho, los fondos captados a sus clientes por esta caja representan, desde esa fecha, la mitad de los del área de la región en la que se inscribe y algo más del 30 por 100 del total de Andalucía.

La importancia que ha conseguido esta caja puede deberse, entre otros factores, a la gestión llevada a cabo por esta entidad de adaptación al entorno y su importante apuesta por las innovaciones tecnológicas (Nieto González et al., 1998).

A la caja almeriense le sigue en importancia la de Granada y a ésta, por orden, las de Sevilla, Huelva, Jaén y Córdoba.

En la zona Occidental, aunque el número de entidades es mayor, el volumen total de recursos ajenos es sensiblemente menor ya que, al ser muchas de ellas de carácter local o comarcal, su dimensión individual es mucho más reducida.

El Cuadro 6 recoge las cifras totales de captación de recursos de las cajas andaluzas y de todo el país. Si bien la actividad ha sido bastante más intensa en la parte oriental, el conjunto de las cajas andaluzas presenta una importante participación en el sector de las cooperativas de crédito españolas que equivale, prácticamente, a una tercera parte del total. La crisis que experimentan estas entidades a comienzos de la década de los ochenta, que afecta de forma muy importante a alguna de las andaluzas, es la causa de que esta participación descienda en 1985 hasta el 21 por 100, para luego, una vez avancen los procesos de saneamiento, ir recuperándose hasta alcanzar de nuevo valores que superan el 30 por 100. Se trata de una cifra realmente excepcional ya que supera al que corresponde a Andalucía en el conjunto español tanto por superficie como por población (Titos Martínez, 2003).

Tabla. 3. Recursos ajenos de las cajas rurales de Andalucía (1975-2000) (Millones de pesetas y porcentajes)

\begin{tabular}{|c|c|c|c|c|c|c|c|}
\hline \multirow{2}{*}{ Año } & \multicolumn{2}{|c|}{ Andalucía Oriental } & \multicolumn{2}{|c|}{$\begin{array}{l}\text { Andalucía } \\
\text { Occidental }\end{array}$} & \multirow{2}{*}{$\begin{array}{c}\text { Total } \\
\text { Andalucía }\end{array}$} & \multirow{2}{*}{$\begin{array}{c}\text { Total } \\
\text { España }\end{array}$} & \multirow{2}{*}{$\begin{array}{c}\% \\
\text { And/Esp }\end{array}$} \\
\hline & Volumen & $\begin{array}{l}\% \text { s/ } \\
\text { And. }\end{array}$ & Volumen & $\begin{array}{l}\% \mathrm{~s} / \\
\text { And. }\end{array}$ & & & \\
\hline 1975 & 9.929 & 43,4 & 12.935 & 56,6 & 22.864 & 75.424 & 30,3 \\
\hline 1980 & 36.490 & 47,1 & 41.018 & 52,9 & 77.508 & 284.884 & 27,2 \\
\hline 1985 & 86.684 & 52,2 & 79.276 & 47,8 & 165.960 & 773.200 & 21,4 \\
\hline
\end{tabular}

23 Al finalizar el siglo serán solo nueve, debido a la absorción de la Caja Rural de La Carlota por parte de la Caja Rural Provincial de Córdoba en 1991.

24 Con la constitución de Cajamar en el año 2000, fruto de la fusión de las Caja de Almería y Málaga, este número se reduce a cuatro. 


\begin{tabular}{rrrrrrrr|}
1990 & 208.693 & 61,1 & 132.758 & 38,9 & 341.451 & 1.218 .725 & 28,0 \\
\hline 1995 & 476.394 & 66,2 & 243.411 & 33,8 & 719.805 & 2.405 .643 & 29,9 \\
\hline 2000 & 1.003 .234 & 74,1 & 350.579 & 25,9 & 1.353 .813 & 4.165 .183 & 32,5
\end{tabular}

Fuente: Elaboración propia con datos de los Anexos 1 y 2 y de Titos Martínez (2003), cuadro 4.03 , pp. $280-281$

Por otro lado, la tendencia general de los recursos captados en ambas zonas es creciente, pero con unas tasas de crecimiento más reducidas en los años de la crisis. Después de una nueva aceleración a mediados de los noventa, el avance se ralentiza de nuevo a finales de siglo. No obstante, la zona Oriental presenta, en media, ritmos de crecimiento más elevados que la Occidental (especialmente debido al impulso de la Caja Rural de Almería) de ahí que el peso de esta zona en el conjunto de la región se vea incrementado de forma tan significativa en estos últimos años del siglo XX (Cuadro 6).

Del análisis de la inversión crediticia de las cajas rurales andaluzas (Anexos 1 y 2) se pueden extraer conclusiones muy similares a las obtenidas en el caso de la captación de recursos de terceros. En primer lugar, la gran superioridad de la Caja Rural Provincial de Almería (y posteriormente de Cajamar). De igual modo, esta es la causa de la primacía del negocio de las entidades de la zona oriental de Andalucía respecto a la occidental. A la caja almeriense le siguen por orden de importancia las cajas provinciales de Granada, Huelva, Sevilla, Jaén y Córdoba. También en este caso, el volumen de recursos invertidos por las cajas locales o comarcales es bastante más limitado que los de las de carácter provincial.

Si tenemos en cuenta el peso que la inversión crediticia de las cajas rurales andaluzas ha tenido en el total del sector a nivel nacional (Cuadro 7), se observa que, al igual que en caso de la captación de recursos, representa aproximadamente una tercera parte del mismo. La crisis de los años ochenta también supuso una caída en esta participación que se recuperará en los años siguientes hasta alcanzar, en el año 2000, algo más del 34 por 100, ligeramente superior a la que marcaba en esa fecha la de recursos de terceros.

En el caso de la financiación otorgada, no hay tanta similitud en la evolución seguida por cada entidad. Si bien, en media, todas han experimentado una trayectoria creciente en el tiempo, hay algunos casos en los que la inversión crediticia se reduce para algunos años. Es el caso, por ejemplo, de la Caja Rural de Jaén que ve como en los primeros años de la década de los ochenta, los recursos destinados a la financiación de clientes se ven reducidos como consecuencia de la importante crisis experimentada por la entidad en esos años. No obstante, en los años siguientes, la caja jiennense se recupera y vuelve a tener tasas de crecimiento en sus operaciones activas similares, o incluso superiores, al resto de las entidades andaluzas. 
Tabla. 4. Inversión crediticia de las cajas rurales andaluzas (1975-2000) (Millones de pesetas y porcentajes)

\begin{tabular}{|c|c|c|c|c|c|c|c|}
\hline \multirow{2}{*}{ Año } & \multicolumn{2}{|c|}{ Andalucía Oriental } & \multicolumn{2}{|c|}{$\begin{array}{l}\text { Andalucía } \\
\text { Occidental }\end{array}$} & \multirow{2}{*}{$\begin{array}{c}\text { Total } \\
\text { Andalucía }\end{array}$} & \multirow{2}{*}{$\begin{array}{l}\text { Total } \\
\text { España }\end{array}$} & \multirow{2}{*}{$\begin{array}{c}\% \\
\text { And/Esp }\end{array}$} \\
\hline & Volumen & $\begin{array}{l}\% \text { s/ } \\
\text { And. }\end{array}$ & Volumen & $\begin{array}{l}\% \text { s/ } \\
\text { And. }\end{array}$ & & & \\
\hline 1975 & 5.371 & 37,6 & 8.901 & 62,4 & 14.272 & 53.795 & 26,53 \\
\hline 1980 & 26.326 & 46,5 & 30.271 & 53,5 & 56.597 & 190.386 & 29,73 \\
\hline 1985 & 52.733 & 54,0 & 44.980 & 46,0 & 97.713 & 437.900 & 22,31 \\
\hline 1990 & 130.323 & 64,1 & 72.860 & 35,9 & 203.183 & 662.337 & 30,68 \\
\hline 1995 & 346.651 & 75,0 & 115.582 & 25,0 & 462.233 & 1.409 .484 & 32,79 \\
\hline 2000 & 925.094 & 77,7 & 265.661 & 22,3 & 1.190 .755 & 3.464 .585 & 34,37 \\
\hline
\end{tabular}

Fuente: Elaboración propia con datos de los Anexos 1y 2 y de Titos Martínez (2003), cuadro 4.05, pp. 284-285.

En cualquier caso, también en esta área de negocio, como en la captación de depósitos, las tasas de crecimiento de la actividad han sido superiores en la zona Oriental que en la Occidental, de ahí que el peso de la primera en el conjunto regional del sector se haya duplicado en este último periodo del siglo (Cuadro 7).

En el Gráfico 1 se recoge la distribución provincial de los recursos de terceros captados por las cooperativas de crédito en Andalucía. Tanto en este caso como en el Gráfico 2 (Distribución provincial de la inversión crediticia), los datos utilizados incluyen, hasta 1987, solo las operaciones de las cajas rurales andaluzas. Desde esa fecha, se tienen en cuenta los datos de todas las cooperativas de crédito con actividad en territorio andaluz, sean cajas rurales o no, con independencia de la localización de su sede social, facilitados por el Banco de España en su Boletín Estadístico.

Del Gráfico 1 se desprende que, a mediados de la década de los setenta, era la provincia de Sevilla la que más recursos aportaba a estas entidades. Sin embargo, diez años después comienza a ser superada por la de Almería, motivado, fundamentalmente, por la fuerte expansión experimentada por la Caja Rural Provincial almeriense. En el año 2000 a esta provincia le siguen, por orden de importancia en cuanto a captación de recursos por estas entidades, Granada, Sevilla, Málaga, Jaén, Huelva, Córdoba y Cádiz.

Ese año, el saldo total del ahorro captado por las cajas rurales andaluzas era de 1,35 billones de pesetas (Cuadro 6) mientras que el registrado por el conjunto de las cooperativas de crédito en esta comunidad asciende a 1,12 billones de pesetas (21,4 por 100 del total a nivel nacional). Esta diferencia puede deberse a que las cifras proceden de fuentes distintas, pero también puede hacer pensar que parte del 
ahorro captado por las cajas rurales de esta región se ha originado fuera de su territorio de origen.

Gráfico. 1. Distribución provincial de los recursos de terceros captados por las cooperativas de crédito en Andalucía (1975-2000).

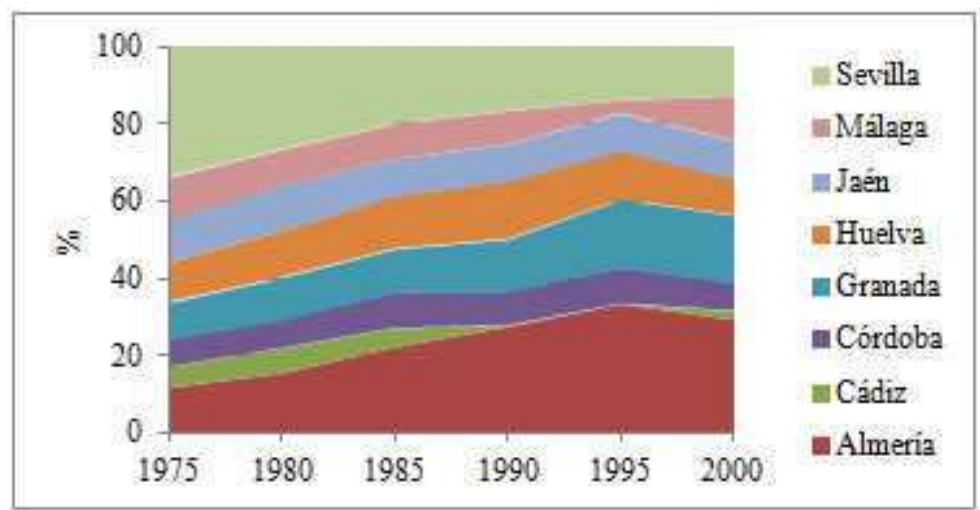

Fuente: Elaboración propia con datos de Titos Martínez (2003), cuadro 4.04, pp. 282 283 y del Boletín Estadístico del Banco de España.

El Gráfico 2 refleja la evolución de la distribución provincial de la inversión crediticia de las cooperativas de crédito en Andalucía. También en este caso, la hegemonía de Sevilla es sustituida por la provincia de Almería. A esta le siguen Granada, Huelva, Málaga, Sevilla, Jaén, Córdoba y Cádiz. De este modo, la provincia granadina se convierte en la segunda en importancia tanto en la captación de recursos como en la inversión crediticia por parte de estas entidades. El conjunto de préstamos de las cooperativas de crédito en Andalucía al finalizar el año 2000 (1,01 billones de euros) representaba aproximadamente un 24 por 100 del total en España, participación ligeramente superior que en el caso de la captación de ahorro. Esta cifra es inferior a la de la inversión crediticia del total de las cajas rurales andaluzas para ese año (1,19 billones de euros - Cuadro 7). Aun considerando que ambos datos proceden de fuentes distintas, se puede pensar que parte de la financiación concedida por las entidades andaluzas se ha destinado a otros territorios fuera de la comunidad autónoma de origen. 
Gráfico. 2. Distribución provincial de la inversión crediticia de las cooperativas de crédito en Andalucía (1975-2000)

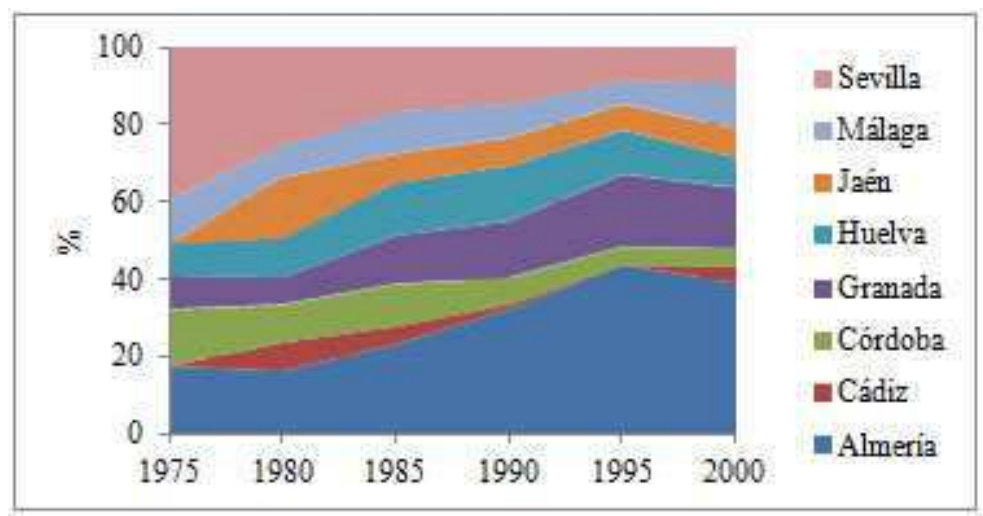

Fuente: Elaboración propia con datos de Titos Martínez (2003), cuadro 4.06, pp. 286287 y del Boletín Estadístico del Banco de España.

Si atendemos a las cuotas de mercado que las cooperativas de crédito alcanzan durante estos años en las provincias andaluzas respecto a bancos y cajas de ahorros (Gráfico 3) se puede decir que, en general, existe una tendencia creciente hasta el año 1995, lo que denota una consolidación del sector en esta región hasta esa fecha. No obstante, en los últimos años del siglo, esta tendencia se ralentiza o incluso experimenta un ligero descenso. Esta trayectoria es común al sector a nivel nacional pero en Andalucía presenta una mayor intensidad. De esta manera, la importancia relativa de las cooperativas de crédito en Andalucía se sitúa, en términos globales, por encima de la media del país, lo que corrobora la relevancia de este tipo de entidades en tierras andaluzas.

En el caso de los depósitos captados, el peso de este conjunto de instituciones en el mercado bancario de ambas partes en las que se ha dividido Andalucía sobrepasa el promedio del país, dado que esto ocurre en todas las provincias excepto en Cádiz. Sin embargo, desde el punto de vista de la inversión crediticia, solo la media de la zona oriental es mayor que la del total a nivel nacional. La mayoría de las provincias de la otra parte de la región (excepto Huelva) sitúan su cuota de mercado en niveles similares al resto del país. De esta manera se puede decir que, aunque en general la importancia relativa de las cooperativas de crédito en Andalucía es superior a la media nacional, lo es con mayor intensidad en el caso de las provincias orientales. Considerando individualmente las provincias, la de Almería destaca con bastante diferencia respecto al resto tanto en el caso de los créditos como de los recursos captados. Le siguen, en ambos casos, Huelva, Granada y Jaén. 
Gráfico. 3. Cuota de mercado de las cooperativas de crédito en las provincias andaluzas (1986-2000).
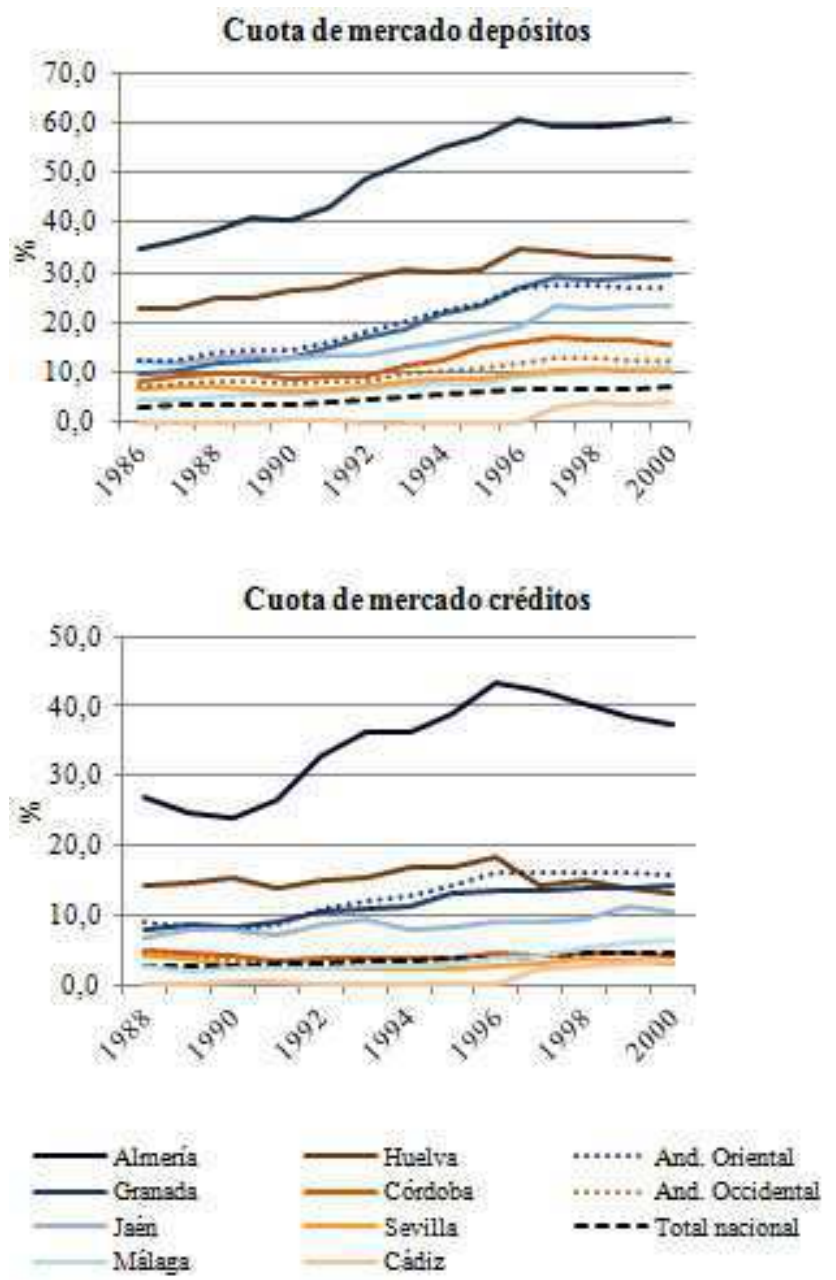

Fuente: Elaboración propia con datos de Titos Martínez (2003), cuadro 4.06, pp. 286287 y del Boletín Estadístico del Banco de España.

Si se analiza la evolución del número de sucursales mantenidas por cada una de las cajas rurales andaluzas (Anexo 3) se observa, de nuevo, la gran expansión experimentada por la Caja Provincial de Almería, especialmente en la década de los noventa, en la que llega a duplicar su ya extensa red de oficinas.

El peso que las oficinas de las cajas rurales con sede en Andalucía (Cuadro 8) representa respecto del total del sector a nivel nacional se ve reducido a mediados de los ochenta como consecuencia de la importante crisis que afectó a estas instituciones. No obstante, pasados esos años, se vuelve a recuperar hasta superar el 31 por 100 en el año 2000 . 
Tabla. 5. Número de oficinas de las cajas rurales andaluzas (1975-2000)

\begin{tabular}{|c|c|c|c|c|c|c|c|}
\hline \multirow[t]{2}{*}{ Año } & \multicolumn{2}{|c|}{$\begin{array}{l}\text { Andalucía } \\
\text { Occidental }\end{array}$} & \multicolumn{2}{|c|}{$\begin{array}{c}\text { Andalucía } \\
\text { Oriental }\end{array}$} & \multirow{2}{*}{$\begin{array}{c}\text { Total } \\
\text { Andalucía }\end{array}$} & \multirow{2}{*}{$\begin{array}{c}\text { Total } \\
\text { España }\end{array}$} & \multirow{2}{*}{$\begin{array}{c}\% \\
\text { And/Esp }\end{array}$} \\
\hline & Oficinas & $\%$ s/ And. & Oficinas & $\%$ s/ And. & & & \\
\hline 1975 & 277 & 54,3 & 233 & 45,7 & 510 & 2.033 & 25,1 \\
\hline 1980 & 308 & 53,8 & 264 & 46,2 & 572 & 2.668 & 21,4 \\
\hline 1985 & 353 & 55,9 & 278 & 44,1 & 631 & 3.350 & 18,8 \\
\hline 1990 & 411 & 61,1 & 262 & 38,9 & 673 & 2.919 & 23,1 \\
\hline 1995 & 533 & 64,6 & 292 & 35,4 & 825 & 3.195 & 25,8 \\
\hline 2000 & 831 & 71,8 & 326 & 28,2 & 1.157 & 3.640 & 31,8 \\
\hline
\end{tabular}

Fuente: Elaboración propia con datos de los cuadros 12 y 13, Anuario Cajas Rurales, Populares y Profesionales (1995 y 2000) y Titos Martínez (2003), cuadro 4.09. pp.292-293.

Los datos sobre el número de oficinas que se han tenido en consideración hasta ahora corresponden al total de sucursales de las cajas rurales andaluzas, tanto en esta región como fuera de ellas. El Gráfico 4 recoge la distribución provincial de las oficinas del conjunto de las cooperativas de crédito en Andalucía, con independencia de la procedencia de las mismas. De nuevo, es la provincia almeriense la que destaca como destino de las oficinas de estas entidades, debido, básicamente, a la presencia de la Caja Rural de Almería. Sin embargo, la diferencia con otras provincias no es tan llamativa teniendo en cuenta la extensa red de esta entidad. Esto puede deberse al hecho de que la caja almeriense y, por consiguiente, la resultante de su fusión con la malagueña, mantenga un número de sucursales fuera de la región bastante considerable. 
Gráfico. 4. Distribución provincial de las oficinas de las cooperativas de crédito en Andalucía 1975-2000



Fuente: Elaboración propia con datos del Boletín Estadístico del Banco de España.

Finalmente, en el Cuadro 9 se registran las cooperativas de crédito que operaban en Andalucía al finalizar el año 2000. De su análisis se pueden señalar algunas conclusiones relevantes. En primer lugar, la extensa red de sucursales con la que contaba en esa fecha la primera de las cajas rurales, tanto dentro como fuera de Andalucía.

Es significativo el hecho de que, además de tener presencia en tres de las provincias andaluzas, posea 263 oficinas (un 46 por 100 de su red) fuera de su región de origen. Esta política de expansión exterior puede deberse, entre otros factores, al hecho de que la provincia almeriense llegó a ser la de mayor grado de saturación de entidades de crédito entre todas las andaluzas, lo que llevó a esta entidad a buscar clientes fuera. En 1996, por ejemplo, en Almería había una oficina por cada 970 habitantes mientras que la media en Andalucía era de una sucursal por cada 1.170 habitantes (Nieto González et al., 1998).

A esta entidad, le siguen por extensión de su red de sucursales, las Cajas Rurales de Granada, Sevilla, Jaén, Huelva y Córdoba, las cuales mantienen una tímida representación de una o dos oficinas fuera de Andalucía. Entre ellas hay algunas con presencia en más de una provincia de la región (Sevilla y Huelva) y otras centradas en su ámbito provincial originario de actuación (Granada, Jaén y Córdoba). A estas entidades les sigue la Caja Rural de Utrera, con cinco oficinas situadas todas en la provincia de Sevilla y, por último, las cajas de ámbito local con una única oficina cada una. 
Tabla. 6. Oficinas de las cooperativas de crédito en Andalucía (Distribución provincial) 31 de diciembre de 2000

\begin{tabular}{|c|c|c|c|c|c|c|c|c|c|c|c|}
\hline & Almería & Cádiz & Córdoba & Granada & Huelva & Jaén & Málaga & Sevilla & $\begin{array}{c}\text { Total } \\
\text { Andalucía }\end{array}$ & $\begin{array}{c}\text { Resto } \\
\text { España }\end{array}$ & $\begin{array}{c}\text { Total } \\
\text { Entidad }\end{array}$ \\
\hline \multicolumn{12}{|c|}{ ENTIDADES CON SEDE EN ANDALUCÍA } \\
\hline Cajamar & 191 & 5 & - & - & - & - & 108 & - & 304 & 263 & 567 \\
\hline $\begin{array}{l}\text { C.R. Prov. de } \\
\text { Granada }\end{array}$ & - & - & - & 138 & - & - & - & - & 138 & 2 & 140 \\
\hline \multicolumn{12}{|l|}{ C.R. Prov. de } \\
\hline C.R. Prov. de Jaén & - & - & - & - & - & 123 & - & - & 123 & 1 & 124 \\
\hline $\begin{array}{l}\text { C.R. Prov. de } \\
\text { Huelva }\end{array}$ & - & 22 & - & - & 81 & - & - & - & 103 & 2 & 105 \\
\hline $\begin{array}{l}\text { C.R. Prov. de } \\
\text { Córdoba }\end{array}$ & - & - & 80 & - & - & - & - & - & 80 & 1 & 81 \\
\hline C.R. Utrera & - & & - & - & - & - & - & 5 & 5 & - & 5 \\
\hline $\begin{array}{l}\text { C.R. Nuestra } \\
\text { Señora Madre del } \\
\text { Sol (Adamuz) }\end{array}$ & - & - & 1 & - & - & - & - & - & 1 & - & 1 \\
\hline $\begin{array}{l}\text { C.R. Nuestra } \\
\text { Señora de } \\
\text { Guadalupe } \\
\text { (Baena) }\end{array}$ & - & & 1 & - & - & - & - & - & 1 & - & 1 \\
\hline $\begin{array}{l}\text { C.R. Nuestra } \\
\text { Señora del Campo } \\
\text { (Cañete de las } \\
\text { Torres) }\end{array}$ & - & - & 1 & - & - & - & - & - & 1 & - & 1 \\
\hline \multirow[t]{2}{*}{$\begin{array}{l}\text { C.R. Nuestra } \\
\text { Señora del } \\
\text { Rosario (Nueva } \\
\text { Carteya) }\end{array}$} & - & - & 1 & - & - & - & - & - & 1 & - & 1 \\
\hline & 191 & 33 & 84 & 138 & 81 & 123 & 108 & 119 & 887 & 270 & 1.157 \\
\hline \multicolumn{12}{|c|}{ ENTIDADES CON SEDE FUERA DE ANDALUCÍA } \\
\hline Caja de Arquitectos & - & - & - & - & - & - & 1 & 1 & 2 & 15 & 17 \\
\hline Caja de Ingenieros & - & & - & - & - & - & - & 1 & 1 & 6 & 7 \\
\hline
\end{tabular}

\begin{tabular}{l|llllllllllll|} 
TOTAL POR & & & & & & & & & & \\
PROVINCIA & 191 & 38 & 84 & 138 & 81 & 123 & 109 & 126 & 890 & 291 & 1.181 \\
\hline
\end{tabular}

C.R. = Caja Rural

Fuente: Elaboración propia con datos del Anuario Cajas Rurales, Populares y Profesionales, 2000.

Por otro lado, cabe señalar la ausencia de cajas rurales foráneas, aunque sí tienen oficina en Andalucía dos cooperativas de crédito no agrarias. De las 15 
oficinas que la Caja de Arquitectos posee en total, dos (13 por 100) se encuentran situadas en territorio andaluz, en concreto, una en Sevilla y otra en Málaga. En el caso de la Caja de Ingenieros, de sus 6 sucursales, una se halla en Sevilla.

Por tanto, al finalizar el siglo XX, las únicas cajas rurales operando en territorio andaluz son las que tienen su sede en esta comunidad autónoma. Mientras que, aunque no existen cooperativas de crédito no agrarias en Andalucía, hay dos de estas entidades, cuya sede social se sitúa en Barcelona, que tienen presencia en la región.

\section{El siglo XXI: crisis económica y concentración del sector. Unas pinceladas}

Los primeros años del nuevo siglo constituyen un periodo muy agitado en el devenir del sistema financiero español. Si bien, durante los primeros años el sistema bancario continúa la gran expansión que se había iniciado en los últimos años de la centuria anterior, a partir de finales del año 2007 se ve inmerso en una de las crisis más importantes de su historia. La magnitud de los problemas puso de manifiesto las debilidades del sistema financiero y de su regulación y supusieron un punto de inflexión en la concepción de los modelos bancarios y de la supervisión del sistema (Vives, 2011).

Cuando se inicia la crisis, el sector bancario español gozaba de una buena situación, con niveles de rentabilidad y solvencia elevados, como consecuencia de la etapa de gran expansión de los años anteriores vinculada, en gran parte, al crecimiento del sector inmobiliario. Sin embargo, esa misma expansión, provocó la generación de una serie de desequilibrios que fueron después los causantes de las dificultades de las entidades de depósito españolas. Entre ellos, cabe señalar el rápido incremento del endeudamiento, tanto a nivel nacional como en el conjunto de las empresas y de las familias, la concentración del negocio bancario en el sector inmobiliario y el aumento de la capacidad instalada, todo ello en un escenario de estabilidad macroeconómica, con abundancia de liquidez y políticas monetarias expansivas (Maudos Villarroya, 2011).

La necesidad de superar una situación de crisis de dimensión internacional y de profundidad poco esperada, llevó a las autoridades españolas a adoptar diversas medidas con el fin de mejorar la solvencia de las entidades y recuperar la confianza en el sistema. Todas estas disposiciones ${ }^{25}$, que se iniciaron en el año 2008, han provocado la más profunda restructuración de sector, que afectó de forma mucho más significativa a las cajas de ahorros que al resto de entidades bancarias. El resultado refleja una intensa concentración de las entidades y, por tanto, una considerable reducción de su número, una importante racionalización de su capacidad instalada así como una mejora en la solvencia y en la liquidez de las entidades (Zurita 2014).

Las cooperativas de crédito también se han visto afectadas por la crisis, aunque de un modo diferente al de las cajas de ahorros. Tal y como señala la Unacc (2013) en el informe sobre la consolidación del sector, el conjunto de las cooperativas de crédito partía de una buena situación con niveles de capitalización elevados, una

25 Una revisión de estas medidas puede verse en Calvo Bernardino y Martín de Vidales (2014). 
amplia base social, un buen nivel de liquidez, una positiva relación entre depósitos y créditos y una morosidad reducida. Sin embargo el sector se ha visto afectado tanto por la intensidad y la duración de la crisis como por los cambios legislativos. Todo ello, unido a la urgencia de aumentar la eficiencia, llevó a estas entidades a iniciar sus propios procesos de concentración, motivados, como ya se ha señalado, más por decisión propia que por obligaciones legales (Fajardo García, 2011).

No obstante, según expone este mismo informe, las nuevas disposiciones legales afectaron a las cooperativas de crédito de una forma menos intensa que a las cajas de ahorros. De un lado, cuando el Real Decreto-Ley 2/2011 para el reforzamiento del sistema financiero español, aumentó los requerimientos mínimos de capital para las entidades bancarias, este sector no tuvo grandes problemas puesto que ya cumplía el 8 por 100 que la nueva norma les exigía ${ }^{26}$. Por otro lado, el Real Decreto-Ley 2/2012, de saneamiento del sistema financiero, además de incrementar la obligación de provisionar los activos inmobiliarios establecía una exigencia adicional de reforzamiento de los fondos propios, en función de esas mismas exposiciones inmobiliarias. De este modo, se planteaban la necesidad de nuevos procesos de reestructuración del sector bancario a efectos de cumplir las exigencias de esta nueva disposición. Sin embargo, mientras que, a partir de entonces, al resto de entidades solo se les permitían procesos de integración mediante fusiones, a las cooperativas de crédito también se les daba la posibilidad de utilizar la fórmula del SIP o del grupo cooperativo, que sólo suponían vínculos contractuales que no implicaban la pérdida de la personalidad jurídica a ninguno de sus miembros.

Todo ello ha permitido al sector de las cooperativas de crédito llevar a cabo su peculiar proceso de concentración de forma tranquila y progresiva, sin necesidad de apoyo público, con una menor exposición al riesgo inmobiliario y cumpliendo los nuevos requerimientos de capital sin dificultad debido a la amplitud de su base societaria (Pizarro-Barceló et al., 2015). Esta transformación se ha materializado a través de dos grandes figuras: las fusiones entre entidades y la creación de grupos cooperativos (con o sin integración en un SIP).

Durante el periodo 2001-2015, las cooperativas de crédito andaluzas se han visto inmersas en estos procesos de concentración ${ }^{27}$. Por lo que se refiere a las fusiones, tres han sido las entidades protagonistas:

Cajamar: entidad que nace en el año 2000 mediante la fusión de las cajas rurales de Almería y Málaga, a las que ya se les había incorporado antes la Caja Rural Campo de Cartagena (1989) y la Caja Grumeco de Madrid (2000). A partir del año 2007 inicia una fase de expansión fuera de su comunidad de origen, especialmente hacia la zona del Levante, integrando hasta ocho cooperativas de crédito no andaluzas: Caja Rural del Duero (2007), Caixa Rural de Balears (2010), Caja Campo (2011), Caja Rural de

26 Las cooperativas de crédito debían mantener un nivel de capital principal superior al 8 por 100 en lugar del 10 por 100 requerido a otras entidades, ya que su financiación no dependía de los mercados mayoristas y mantenían una amplia base social (o accionarial), por lo que no se les aplicaban los requerimientos adicionales El proceso de concentración del sector de las cooperativas de crédito en los primeros años del siglo XXI se recoge en Belmonte Ureña (2011), Palomo Zurdo y Gutiérrez Fernández (2012) y UNACC (2013). 
Castellón San Isidro y Ruralcaja ${ }^{28}$ (2012) y, por último la Caja Rural de Canarias, Crèdit Valencia y Caja Rural Casino (2013). Cuando en 2012 se fusionan Cajamar y Ruralcaja, la denominación social pasa a ser Cajas Rurales Unidas. No obstante, en el año 2014 Cajamar Caja Rural decide liquidar esta marca comercial y seguir usando la que estableció desde su constitución en el año 2000.

- Caja Rural del Sur: entidad resultante de la fusión, en el año 2001, de las cajas rurales provinciales de Sevilla y Huelva. Posteriormente, en 2014, se integraría también la Caja Rural de Córdoba.

- Caja Rural de Jaén, Madrid y Barcelona integra a finales de 2015 a la madrileña Novanca (Caja de Crédito Cooperativo) mediante una operación de fusión por absorción de la primera sobre la segunda. De este modo, la entidad jiennense asume la red de oficinas de la absorbida formada por 18 sucursales en el sur de Madrid.

Desde el punto de vista de la formación de grupos cooperativos, las cajas rurales andaluzas se han visto inmersas en varios procesos que se resumen en el Cuadro 10:

Tabla. 7. Las cajas rurales andaluzas y la formación de grupos cooperativos (2009-2013)

\begin{tabular}{ccc} 
Grupo Cooperativo & $\begin{array}{c}\text { Fecha de } \\
\text { constitución }\end{array}$ & $\begin{array}{c}\text { Entidad cabecera e integrantes a } 31 \text { de } \\
\text { diciembre de 2013 }\end{array}$ \\
\hline
\end{tabular}

\begin{tabular}{|c|c|c|}
\hline G. C. Cajamar $^{(1)}$ & 2009 & Cajamar Caja Rural $^{(2)}$ \\
\hline & & Caixa Rural Altea \\
\hline & & Caixa Rural de Albalat dels Sorells \\
\hline & & Caixa Rural de Callosa d'en Sarrià \\
\hline & & Caixa Rural de Turís \\
\hline & & Caixa Rural San Josep de Vilavella \\
\hline & & Caixa Rural Torrent \\
\hline & & Caja de Crédito de Petrel, Caja Rural \\
\hline & & Caja Rural Católico Agraria \\
\hline & & Caja Rural de Cheste \\
\hline & & Caja Rural de Villar \\
\hline & & Caja Rural del Alginet \\
\hline & & Caja Rural La Junquera de Chilches \\
\hline & & Caja Rural san Isidro de Vilafamés \\
\hline
\end{tabular}

28 Caja Rural del Mediterráneo - Ruralcaja, a su vez, se constituye en 2001 por la integración de la Caja Rural de Valencia, la Caja Rural de Alicante y Credicoop en Castellón. A este proyecto se unirá la Caja Rural de Elche, en 2004. 
Caja Rural San Jaime de Alquerías Niño Perdido

Caja Rural San José de Burriana

Caja Rural San José de Nules

Caja Rural San Roque de Almenara

Caja Rural San Vicente Ferrer de la Vall d’Uixó

G. C. Ibérico de Crédito

(3) 2011

Caja Rural del Sur

Caja Rural de Extremadura

Caja Rural de Córdoba

G.C. Solventia 2011 Caja Rural de Almendralejo

Caja Rural de Baena Ntra. Sra. de Guadalupe Caja Rural de Cañete de las Torres Ntra. Sra. del Campo

Caja Rural de Utrera

Caja Rural Ntra. Madre del Sol (Adamuz)

Caja Rural Ntra. Sra. del Rosario (N. Carteya)

(En negrita las entidades cabecera de los grupos)

(1) Entre 2012 y 2013, bajo la denominación Grupo Cooperativo Cajas Rurales Unidas.

(2) Entre 2012 y 2013, bajo la denominación Cajas Rurales Unidas, Sociedad Cooperativa de Crédito.

${ }^{(3)}$ Disuelto en 2014.

Fuente: UNACC, Anuario Estadístico de las Cooperativas de Crédito (2014)

Sin embargo, en el año 2014 se producen algunos cambios en esta configuración. El Banco de España aprueba la disolución del Grupo Cooperativo Ibérico ante el deseo de la Caja de Extremadura de abandonar el proyecto, lo que facilita la fusión de las otras dos entidades que componían dicho grupo: Caja Rural del Sur y Caja Rural de Córdoba, absorbida por la primera.

Por otro lado, en enero de ese mismo año 2014, a iniciativa de Cajamar, se constituye el Banco de Crédito Cooperativo (BCC). Las entidades que participan en este proyecto son un total del 32. Por un lado, las 19 instituciones que integran el Grupo Cooperativo Cajamar y, por otro, trece cajas rurales de Andalucía, Castilla-La Mancha, Cataluña, Comunidad Valenciana y Extremadura ${ }^{29}$. Todas ellas, formen parte o no del Grupo Cajamar, son accionistas del nuevo Banco, siendo la participación más significativa la de la propia entidad almeriense.

29 Estas trece entidades son: Caja Rural de Castilla-La Mancha, Caixa Guissona, las cajas rurales de La Vall d’Uixó, Almassora, Benicarló, Vinaròs y Les Coves de Vinroma, además de las seis entidades que componen el Grupo Solventia. 
Una vez constituido, el BCC se responsabiliza de la dirección y representación del Grupo Cooperativo Cajamar, asumiendo la condición de entidad cabecera del mismo.

Por último, cabe señalar la intención, puesta de manifiesto por el gobierno nacional en el año 2014, de reformar la regulación del sector de las cooperativas de crédito, con el objetivo de avanzar en el proceso de reestructuración de este grupo de entidades. Sin embargo, estos cambios no se van a acometer tan pronto como estaba previsto en un principio aunque, en un futuro, podrían suponer nuevas transformaciones en el sector (Gonzalo Alconada, 2015).

\section{Conclusiones}

Las primeras cooperativas de crédito surgen en España a finales del siglo XIX con algo de retraso respecto a otros países del centro de Europa. Las experiencias iniciales de estas entidades estuvieron vinculadas, casi exclusivamente, al ámbito agrario. Estos planteamientos llegaron con algo de retraso a Andalucía donde las primeras referencias a este tipo de entidades corresponden a los primeros años del siglo XX. Se trataba de cajas rurales vinculadas a sindicatos agrícolas, muy frecuentes en aquella época.

Después de una fase de crecimiento, frenada por la Guerra Civil, el sector experimenta un proceso doble hasta mediados de la década de los setenta. Por un lado, una importante expansión en cuanto al número de entidades y por otro una tendencia a la integración de entidades, tanto por las absorciones de las de ámbito local por parte las de carácter provincial, como por las liquidaciones y disoluciones. En el Registro de Cooperativas de Crédito de la Junta de Andalucía hay referencias sobre más de treinta cooperativas de crédito que surgen por esos años. No obstante, de todas ellas solo 21 llegan a inscribirse como tales en el Registro Oficial de Entidades del Banco de España. Es muy probable que, el resto fueran tan solo secciones de crédito de otras cooperativas agrícolas (que por aquel entonces también se denominaban cajas rurales) que, o bien no consiguen la autorización por parte del Banco de España para operar de forma autónoma, o bien se integran en las cajas rurales de su correspondiente ámbito provincial.

En el caso de Andalucía, se puede decir que entre 1957, fecha de constitución de la Caja Rural Provincial de Jaén, y 1970, año de fundación de la Caja Rural Provincial de Granada, se delimita la configuración "moderna" del sector de las cooperativas de crédito de la región.

Entre esas dos fechas, se fundan un total de 21 cooperativas de crédito. De todas ellas, tan sólo cuatro no tienen carácter agrícola. Se trata de otro tipo de entidades que no perdurarían, al desaparecen por disolución o absorción por parte de otras antes de 1980. Las 17 restantes, son cajas rurales de ámbitos diversos: local, comarcal y provincial. Por las mismas causas anteriores, algunas desparecen del panorama financiero andaluz antes de finalizar el siglo XX. En concreto, en el año 2000 , tenían su sede en este territorio un total de 12 cajas rurales, dos de las cuales (Cajas Rurales de Almería y Málaga) se integran ese mismo año en una nueva entidad, Cajamar. 
Del análisis de la actividad de las cajas rurales andaluzas durante el último cuarto del siglo XX se pueden extraer varias conclusiones:

a) Una vez que las cooperativas de crédito superan las consecuencias de la importante crisis experimentada por el sector en los primeros años de la década de los ochenta, se inicia una fase expansiva que supone la consolidación de este grupo de entidades en el panorama bancario andaluz.

Por un lado, el peso que las cooperativas de crédito andaluzas representan respecto al total nacional se incrementa tanto en el caso de los créditos concedidos y de los recursos captados como del número de oficinas, llegando a superar el 30 por100 del mismo en los tres casos.

Por otro lado, el hecho de que la cuota de mercado (tanto de depósitos como de inversión crediticia) de estas entidades en Andalucía supere durante este periodo a la media para el total del país, apoya igualmente el fortalecimiento de este grupo de entidades. De hecho, dado que estas cuotas alcanzan en la región un máximo en 1995, se puede decir que esa consolidación se produce básicamente hasta esa fecha.

b) En conjunto, las cooperativas de crédito de la zona oriental de la región superan a las de la occidental tanto en volumen de créditos y depósitos como en número de sucursales, a pesar de que el número de instituciones en significativamente inferior. Además esta diferencia se ha ido agrandando con los años. Las causas se pueden centrar en dos aspectos: la presencia de la Caja Rural de Almería (después Cajamar, cooperativa de crédito de mayor dimensión en España) en la parte oriental y la existencia de un buen número de pequeñas entidades de carácter local en la occidental y, por tanto, de menor dimensión.

c) Desde el punto de vista territorial, la presencia y la actividad de las cajas rurales andaluzas, se ha ido desviando de las provincias occidentales (especialmente Sevilla) hacia las orientales (fundamentalmente Almería). No obstante, desde el punto de vista de la localización de oficinas, este trasvase ha sido de menor intensidad, lo que concuerda con una mayor presencia fuera de la región de la entidad almeriense.

d) Consideradas individualmente, el principal rasgo a destacar entre las cajas rurales andaluzas es la heterogeneidad en el tamaño. Entre todas ellas, destaca, con diferencia, la Caja Rural Provincial de Almería que, antes de su fusión con la de Málaga, representaba algo más del 30 por 100 del total de los recursos captados y del 40 por100 de la inversión crediticia de estas entidades en Andalucía. Una vez integradas las dos entidades, la sociedad resultante, Cajamar, se convertirá en la cooperativa de crédito más importante por tamaño, no solo a nivel andaluz sino también a escala nacional.

Finalmente, cabe señalar que tras los procesos de concentración y de expansión geográfica experimentados por estas entidades en los últimos años del siglo XX, el sector de las cooperativas de crédito en Andalucía quedaba conformado por 12 cajas rurales con sede en la región (con 887 oficinas) y otras dos cooperativas de crédito con agrícola foráneas (con tres oficinas). Las entidades andaluzas, habían comenzado también su expansión tanto operativa como presencial en otras zonas geográficas, de ahí que, al finalizar el año 2000, mantuvieran un total de 270 sucursales fuera de esta comunidad autónoma. 
No obstante, en el los comienzos del siglo XXI, esta configuración se alterará como consecuencia de los procesos de concentración del sector, intensificados a raíz de la crisis a la que habrá de hacer frente el sistema bancario español.

\section{Referencias bibliográficas}

BANCO DE ESPAÑA (1975-2000) Boletín Estadístico, Instituciones financieras, Información obtenida de los Estados de Supervisión. http://www.bde.es/bde/es/secciones/informes/boletines/Boletin_Estadist/

BANCO DE ESPAÑA Registro Oficial de Entidades, Consulta de datos históricos de las entidades. http://app.bde.es/ren/html/inicio.htm

Belmonte Ureña, L.J. (2007) El sector de cooperativas de crédito en España: un estudio por comunidades autónomas. Sevilla: Consejo Económico y Social de Andalucía.

Belmonte Ureña, L.J. (2011) El nuevo modelo de negocio de las cooperativas de crédito españolas. Perspectivas futuras, Papeles de Economía Española, $\mathrm{N}^{\circ}$ 130, pp. 244-257.

Bernal, A.M. (1991) Pósitos, cillas, tercias y economía agraria en Andalucía. En: Gil Pérez, M.D.; Torres Hidalgo, M. y Ramírez Moreno, N. (eds.), Pósitos, cillas y tercias en Andalucía. Catálogo de antiguas edificaciones para almacenamiento de grano. Sevilla: Consejería de Obras Públicas y Transportes. Dirección General de Arquitectura. Junta de Andalucía, pp. 51-73.

Calvo Bernardino, A. y Martín de Vidales Carrasco, I. (2014) Crisis y cambios estructurales en el sector bancario español: Una comparación con otros sistemas financieros. Estudios de Economía Aplicada, Vol. 32, № 2, pp. 535-566.

Carasa Soto, P. (1983) Los pósitos en España en el siglo XIX. Investigaciones Históricas: Época Moderna y Contemporánea, $\mathrm{N}^{\circ}$ 4, pp. 247-304.

Carbonell de Masy, R. (1973) Cooperativas de crédito-no rural en Andalucía: ¿son instituciones necesarias para su desarrollo?. Estudios Cooperativos, $\mathrm{N}^{\circ} 30$, pp. 71-77.

Conde Rodríguez, C. (1995) Las cooperativas de crédito en España. REVESCO. Revista de Estudios Cooperativos, N 61, pp. 127-140.

Fajardo García, G. (2011) How viable are spanish credit cooperatives after recent bank capitalization and restructuring regulations?. CIRIEC-España, Revista De Economía Pública, Social Y Cooperativa, No 73 , pp. 151-170.

Gámez Amián, A. (1998) El crédito institucional a la agricultura en Andalucía (17681936). Málaga: Universidad de Málaga.

Garrido Herrero, S. (1995) El cooperativismo agrario español del primer tercio del siglo XX. Revista de Historia Económica, Vol. 13, № 1, pp. 115-144.

González Alcón, J.M. (1995) Las Cajas Rurales en España: una aproximación a la realidad andaluza. Boletín Económico de Andalucía, $\mathrm{N}^{\circ}$ 19, pp. 221-231.

Gonzalo Alconada, Á. (2015) Economía retrasa la reforma de las cooperativas de crédito., Cinco Días (edición digital), 27-02-2015, http://cincodias.com/cincodias/2015/02/26/mercados/1424983200_401591.html

Gutiérrez Fernández, M., Palomo Zurdo, R. y Romero Cuadrado, M. (2012) La expansión territorial como factor motivador de la reestructuración del sistema financiero español: el caso de las cajas de ahorro y las cooperativas de crédito. REVESCO. Revista de Estudios Cooperativos, № 107, pp. 7-34. DOI: 10.5209/rev_REVE.2012.v107.38746

Hidalgo Fernández, A. y Bernabéu Aguilera, F.J. (2014) Un análisis de la regulación andaluza de secciones de crédito. REVESCO. Revista De Estudios Cooperativos, $\mathrm{N}^{\circ}$ 114, pp. 59-83. DOI: 10.5209/rev_REVE.2014.v114.44289. 
López Yepes, J. (2003) Francisco Rivas Moreno: la obra de un reformador social. Migueturra, 1951 - Madrid, 1935. $2^{\text {a }}$ ed. Miguelturra (Ciudad Real): Ayuntamiento de Miguelturra.

Luque Frías, J. (2012) Crisis económica y cooperativas de crédito: el reto de la eficiencia. Tesis doctoral. Departamento de Economía Internacional y de España. Universidad de Granada.

Martín Mesa, A. (1986) Crisis y saneamiento de la Caja Rural Provincial de Jaén. Boletín de la Cámara Oficial De Comercio e Industria de Jaén, № 42, pp. 16-27.

Martín Mesa, A. (1987) Un nuevo modelo orgánico de las Cajas Rurales españolas: especial referencia a las andaluzas. Revista de Estudios Regionales, № 17, pp. 71-102.

Martín Mesa, A. (1988) La crisis de las cajas rurales españolas y el nuevo modelo del cooperativismo del crédito agrario. Granada: Banco de Crédito Agrícola - Instituto de Desarrollo Regional de la Universidad de Granada.

Martín Zamora, M.P. (2000) La solvencia en las cajas rurales provinciales andaluzas (1978-1985): un análisis mediante sistemas de inducción de árboles de decisión. Huelva: Universidad de Huelva.

Martínez Rodríguez, S. y Martínez Soto, Á.P. (2008) Los pioneros del cooperativismo agrario de crédito español (1880-1920). CIRIEC-España, Revista de Economía Pública, Social y Cooperativa, № 63, pp. 89-112.

Martínez Soto, Á.P. (1994) El papel del crédito y la financiación en la agricultura capitalista (1850-1970): Una primera aproximación a un campo multifactorial. Noticiario de Historia Agraria, Vol. 4, №. 7, pp. 39-66.

Martínez Soto, Á.P. (2000) Cooperativismo y crédito agrario en la Región de Murcia, 18901936. Historia Agraria, No 20, pp. 123-167.

Martínez Soto, Á.P. (2003a) Los orígenes del cooperativismo de crédito agrario en España, 1890-1934. CIRIEC-España, Revista de Economía Pública, Social y Cooperativa, № 44, pp. 57-104.

Martínez Soto, Á.P. (2003b) El cooperativismo de crédito en España, 1890-1934. Modelos, sistemas de gestión y balance de su actuación. Historia Agraria, № 30, pp. 119-150.

Martínez Soto, Á.P. (2007) Los pósitos en el siglo XIX: una red pública de microcrédito agrario (1800-1914). Historia Agraria, № 43, pp. 485-530.

Maudos Villarroya, J. (2011) El sector bancario español en el contexto internacional: El impacto de la crisis. Madrid: Fundación de las Cajas de Ahorros.

Melián Navarro, A. (2004) El fenómeno concentratorio como estrategia de crecimiento en las cajas rurales españolas. El Grupo Caja Rural. REVESCO. Revista de Estudios Cooperativos, $\mathrm{N}^{\mathrm{o}} 82$, pp. 89-112.

Melián Navarro, A., Palomo Zurdo, R.J., Sanchis Palacio, J.R. y Soler Tormo, F. (2006) La investigación en materia de cooperativas de crédito y de g rupos cooperativos. CIRIECEspaña, Revista de Economía Pública, Social y Cooperativa, № 56, pp. 25-64.

Nieto González, F.J., Belmonte Ureña, L.J. y Nieto González, J.L. (1998) Las Cajas Rurales en Andalucía en el período 1990-1996: Evolución y situación. Revista de Humanidades y Ciencias Sociales, № 16, pp. 81-108.

Noguer, N. (1980) Las cajas rurales en España y en el extranjero. Granada: Alhacaba. (Original publicado en 1912).

Palomo Zurdo, R.J. (1995) La banca cooperativa en Europa. Madrid: Unión Nacional de Cooperativas de Crédito y Asociación Española de Cooperativas de Crédito.

Palomo Zurdo, R.J. (2004). El crédito cooperativo español en el marco de la UEM: crecimiento y expansión territorial. Mediterráneo Económico, № 6, pp. 110-132.

Palomo Zurdo, R. y Gutiérrez Fernández, M. (2012) El desajuste del crédito en el sistema bancario y la acción de la economía social: el camino de la reestructuración. REVESCO: Revista de Estudios Cooperativos, $\mathrm{N}^{\mathrm{o}} 109, \quad$ pp. 138-169. http://dx.doi.org/10.5209/rev_REVE.2012.v109.40653 
Palomo Zurdo, R.J. y Mateu Gordon, J.L. (2013) Las sociedades cooperativas de crédito en España: antecedentes históricos, evolución y adaptación estratégica ante la crisis financiera. En: G. Lejarriaga Pérez de las Vacas, S. Martín López y A. Muñoz García (eds.), 40 años de historia de las empresas de participación. Madrid: Escuela de Estudios Cooperativos de la Universidad Complutense de Madrid, Editorial Verbum, pp. 502-526.

Palomo Zurdo, R.J. y Sanchis Palacio, J.R. (2008) Un análisis del crédito cooperativo en España: situación actual, expansión territorial y proyección estratégica. Estudios de Economía Aplicada, Vol. 26, Nº 1, pp. 27-41.

Palomo Zurdo, R.J. y Sanchis Palacio, J.R. (2010) Efectos de las fusiones sobre la concentración y la eficiencia bancaria: el caso de las Cajas Rurales y los retos de la crisis financiera. Revista Española de Financiación y Contabilidad, No. 146, pp. 289319.

Palomo Zurdo, R.J. y Valor Martínez, C. (2001) Banca cooperativa: entorno financiero y proyección social. Madrid: Unión Nacional de Cooperativas de Crédito.

Palomo Zurdo, R.J., Sanchis Palacio, J.R. y Gutiérrez Fernández, M. (2011). Efectos de la crisis financiera sobre la innovación en la reorganización de los sistemas bancarios: los sistemas institucionales de protección en las entidades financieras de ámbito territorial. Innovar: Revista de Ciencias Administrativas y Sociales, Vol. 21, № 39, pp. 179-190.

Pizarro-Barceló, R., García-Ortiz, Á y Lópaz-Pérez, A. (2015) Asimetrías en las cooperativas de crédito españolas. CIRIEC-España, Revista De Economía Pública, Social y Cooperativa, $\mathrm{N}^{\mathrm{o}} 84$, pp. 101-131.

Pons, M.Á. (2011) Las principales reformas del sistema financiero español. En J. L. Malo de Molina y P. Martín Aceña (Eds.), Un siglo de historia del sistema financiero español. Madrid: Alianza Editorial, pp. 87-115.

Pueyo Sánchez, J. (2003) Oligopolio y competencia en la banca española del siglo XX: concentración económica y movilidad intra-industrial, 1922-1995. Revista de Historia Económica, Vol. 21, № 1, pp. 147-195.

Rivas Moreno, F. (1895) El crédito agrícola y el ahorro. Toledo: Imprenta, librería y encuadernación de Menor Hermanos. Digitalizado por el Proyecto Rivas Moreno, Centro de Estudios de Castilla-La Mancha, Universidad de Castilla-La Mancha.

http://www.uclm.es/ceclm/rivasmoreno/Libros/credito_agricola_ahorro/index.htm

Rivas Moreno, F. (1902) Las cajas rurales, Granada: Imprenta El Defensor de Granada. Digitalizado por el Proyecto Rivas Moreno, Centro de Estudios de Castilla-La Mancha, Universidad de Castilla-La Mancha.

http://www.uclm.es/ceclm/rivasmoreno/Libros/cajas_rurales/pages/00000.htm

Rodero Franganillo, A. (1974) Las cajas rurales españolas. Madrid: Ediciones ICE.

Rodero Franganillo, A. (1980) Las cajas rurales andaluzas. Un instrumento financiero para el desarrollo. Revista de Estudios Regionales, Vol. II, No Extra, pp. 481-501.

Sánchez Antonijuan, E. (1985) Las cajas rurales como primer intermediario del sector agrario andaluz. Revista de Estudios Andaluces, № 5, pp. 73-98.

Sánchez Picón, A. y Navarro Pérez, L.C. (2001) Algunas cuestiones en torno al crédito agrario en Andalucía (1800-1936). Areas: Revista Internacional de Ciencias Sociales, No 21 , pp. 165-184.

Server Izquierdo, R.J. y Melián Navarro, A. (2001) Evaluación de la eficiencia de las entidades financieras en las secciones de crédito de las cooperativas. Investigación Agraria. Producción y Protección Vegetales, Vol. 16, № 1, pp. 87-104.

Soler Tormo, F.V. (2002) La identidad cooperativa como garantía de futuro: Las cooperativas de crédito ante la trivialización de sus principios. CIRIEC-España, Revista de Economía Pública, Social y Cooperativa, No 40, pp. 215-242. 
Tello Aragay, E. (1994) El papel del crédito rural en la agricultura del Antiguo Régimen: desarrollo y crisis de las modalidades crediticias (1600-1850). Noticiario de Historia Agraria, $\mathrm{N}^{\mathrm{o}}$ 7, pp. 9-37.

Terrón Muñoz, F. (1987) Las Cajas rurales españolas: nacimiento, auge y perspectivas del cooperativismo agrario crediticio en España. Granada: Universidad de Granada.

Titos Martínez, M. (2003) El sistema financiero en Andalucía: tres siglos de historia (1740-2000). Sevilla: Instituto de Estadística de Andalucía.

Titos Martínez, M. (2005) Historia de la Caja Rural de Granada (1970-2005). Granada: Fundación Caja Rural de Granada.

UNACC (1973, 1974) Anuario Estadístico de las Cooperativas de Crédito. Madrid

UNACC (1980) Anuario Estadístico Cajas Rurales y Cooperativas de Crédito. Madrid.

UNACC (1985) Anuario de las Cooperativas de Crédito y Cajas Rurales. Madrid.

UNACC (1995) Anuario Cajas Rurales, Populares y Profesionales. Madrid.

UNACC (2000) Anuario Cajas Rurales, Populares y Profesionales. http://www.unacc.com/Portals/0/Anuario/Anuarios\%20Anteriores/anuario2000.pdf

UNACC (2013) Las cooperativas de crédito, la consolidación dentro del modelo, Informe sobre la consolidación del sector, datos a diciembre de 2013. http://www.unacc.com/Portals/0/Otras\%20Publicaciones/Libros/Reestructuraci\%C3\%B $\underline{3 \mathrm{n} \% 202013 . \mathrm{pdf}}$

UNACC (2014) Anuario Estadístico de las Cooperativas de Crédito. http://www.unacc.com/Portals/0/Anuario/Anuario/2014\%20newsletter/ANUARIO\%20 $\underline{2014 . p d f}$

Vargas Vasserot, C. V. (2015). Las cooperativas de crédito y su posición dentro del modelo cooperativo. Integración frente a diferenciación en el marco de la reforma del sistema financiero. REVESCO. Revista de Estudios Cooperativos, No 117, pp. 50-76. DOI: 10.5209/rev_REVE.2015.v117.48145.

Vives, X. (2011) La industria financiera española en el inicio del siglo XXI: situación y retos de futuro. En: Malo de Molina, J.L. y Martín Aceña, P. (eds.), Un siglo de historia del sistema financiero español. Madrid: Alianza Editorial, pp. 415-456.

Zurita, J. (2014) La reforma del sector bancario español hasta la recuperación de los flujos de crédito. BBVA Research. Documento de Trabajo No 14/12. 


\section{ANEXO 1 RECURSOS AJENOS E INVERSIÓN CREDITICIA DE LAS CAJAS RURALES DE ANDALUCÍA ORIENTAL (1975-2000)}

(Millones de pesetas)

\section{RECURSOS AJENOS}

\begin{tabular}{rrrrrrrr}
\hline Año & $\begin{array}{c}\text { C.R. } \\
\text { Prov. } \\
\text { Almería }\end{array}$ & $\begin{array}{c}\text { C.R. } \\
\text { Prov. } \\
\text { Málaga }\end{array}$ & Cajamar & $\begin{array}{c}\text { C.R. } \\
\text { Prov. } \\
\text { Granada }\end{array}$ & $\begin{array}{c}\text { C.R. } \\
\text { Prov. } \\
\text { Jaén }\end{array}$ & $\begin{array}{c}\text { C.R. San José } \\
\text { (Torredonjimeno) }\end{array}$ & $\begin{array}{c}\text { Total } \\
\text { Andalucía } \\
\text { Oriental }\end{array}$ \\
\hline & & & & & & & \\
1975 & 2.652 & 2.514 & - & 2.188 & 2.575 & - & 9.929 \\
1980 & 11.786 & 7.022 & - & 8.348 & 9.277 & 57 & 36.490 \\
1985 & 36.906 & 14.680 & - & 18.451 & 16.474 & 173 & 86.684 \\
1990 & 102.095 & 29.282 & - & 44.877 & 32.439 & - & 208.693 \\
1995 & 234.076 & 54.731 & - & 120.096 & 67.491 & - & 476.394 \\
\hline 2000 & - & -689.311 & 198.264 & 115.659 & - & 1.003 .234 \\
\hline
\end{tabular}

\section{INVERSIÓN CREDITICIA}

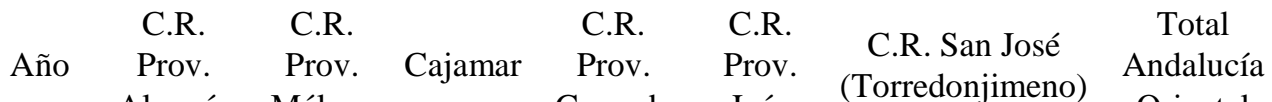

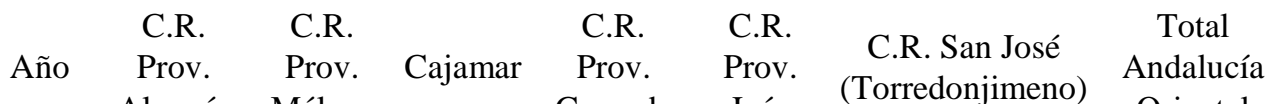



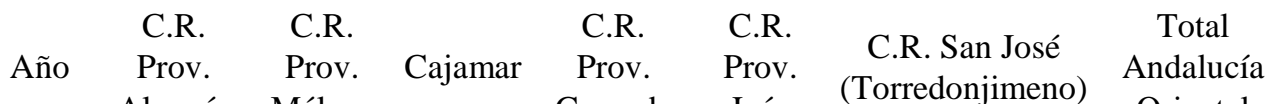

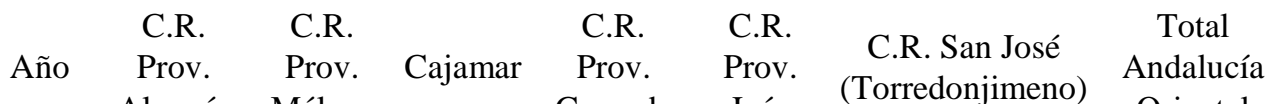

Almería Málaga Granada Jaén (Torredonjimeno) Oriental

\begin{tabular}{rrrrrrrrr}
\hline & & & & & & & \\
1975 & 2.491 & 1.604 & - & 1.276 & & 5.371 \\
1980 & 9.069 & 4.498 & - & 3.926 & 8.808 & 25 & 26.326 \\
1985 & 22.627 & 10.647 & - & 12.302 & 7.125 & 32 & 52.733 \\
1990 & 69.410 & 16.899 & - & 29.587 & 14.427 & - & 130.323 \\
1995 & 201.843 & 26.657 & - & 88.111 & 30.040 & - & 346.651 \\
\hline 2000 & - & -679.426 & 166.876 & 78.792 & - & 925.094 \\
\hline
\end{tabular}

Fuente: Elaboración propia con datos de Anuario Estadístico Cajas Rurales y Cooperativas de Crédito (1980), Anuario de las Cooperativas de Crédito y Cajas Rurales (1985), Anuario

Cajas Rurales, Populares y Profesionales (1995 y 2000) y Titos Martínez (2003), cuadro 4.05 , pp. $284-285$. 


\section{ANEXO 2 RECURSOS AJENOS E INVERSIÓN CREDITICIA DE LAS CAJAS RURALES DE ANDALUCÍA OCCIDENTAL (1975-2000)}

(Millones de pesetas)

\section{RECURSOS AJENOS}

\begin{tabular}{|c|c|c|c|c|c|c|c|c|c|c|c|}
\hline Año & $\begin{array}{l}\text { C.R. } \\
\text { Prov. } \\
\text { Cádiz }\end{array}$ & $\begin{array}{c}\text { C.R. } \\
\text { Prov. } \\
\text { Córdoba }\end{array}$ & $\begin{array}{c}\text { C.R. N } \mathrm{N}^{\mathrm{a}} \\
\mathrm{S}^{\mathrm{a}} \text { Madre } \\
\text { del Sol } \\
\text { (Adamuz) }\end{array}$ & $\begin{array}{l}\text { C.R. } \mathrm{N}^{\mathrm{a}} \mathrm{S}^{\mathrm{a}} \\
\text { de } \\
\text { Guadalupe } \\
\text { (Baena) }\end{array}$ & $\begin{array}{c}\text { C.R. } N^{\mathrm{a}} \\
\mathrm{S}^{\mathrm{a}} \text { del } \\
\text { Campo } \\
\text { (Cañete } \\
\text { de las } \\
\text { Torres) } \\
\end{array}$ & $\begin{array}{c}\text { C.R. } \\
\text { La } \\
\text { Carlota }\end{array}$ & $\begin{array}{c}\text { C.R. } \mathrm{N}^{\mathrm{a}} \\
\mathrm{S}^{\mathrm{a}} \text { del } \\
\text { Rosario } \\
\text { (Nueva } \\
\text { Carteya) }\end{array}$ & $\begin{array}{l}\text { C.R. } \\
\text { Prov. } \\
\text { Huelva }\end{array}$ & $\begin{array}{c}\text { C.R. } \\
\text { Prov. } \\
\text { Sevilla }\end{array}$ & $\begin{array}{l}\text { C.R. } \\
\text { Utrera }\end{array}$ & $\begin{array}{c}\text { Total } \\
\text { Andalucía } \\
\text { Occidental }\end{array}$ \\
\hline 1975 & 1.236 & 1.633 & - & - & - & - & - & 2.305 & 7.761 & - & 12.935 \\
\hline 1980 & 5.048 & 5.546 & - & 171 & - & 47 & - & 9.147 & 20.613 & 446 & 41.018 \\
\hline 1985 & 8.199 & 13.980 & 427 & 589 & 381 & a 351 & a 277 & 22.355 & 33.345 & ${ }^{\mathrm{a}} 1.118$ & 79.276 \\
\hline 1990 & - & 25.424 & 733 & 989 & 691 & 552 & 746 & 49.740 & 53.883 & ${ }^{b} 2.582$ & 132.758 \\
\hline 1995 & - & 53.773 & 2.234 & 2.623 & 1.901 & - & 1.743 & 86.061 & 90.460 & 4.616 & 243.411 \\
\hline 2000 & - & 69.845 & 2.215 & 3.555 & 2.884 & - & 2.529 & 125.253 & 137.541 & 6.757 & 350.579 \\
\hline
\end{tabular}

\section{INVERSIÓN CREDITICIA}

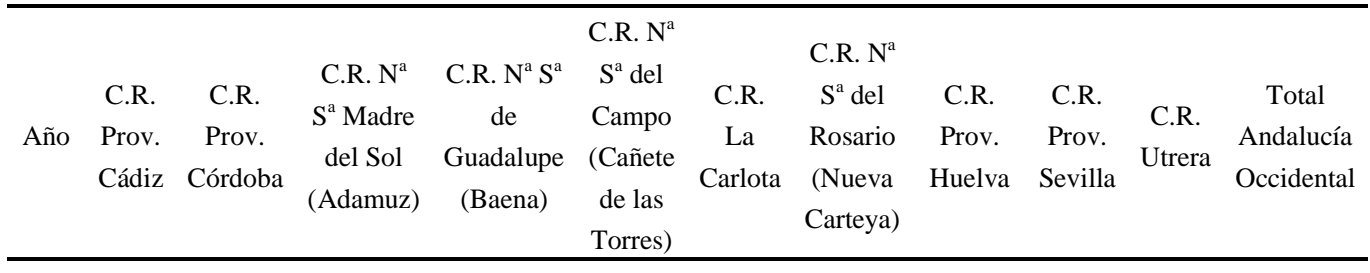

\begin{tabular}{rrrrrrrrrrrr}
1975 & & 2.095 & - & - & - & - & - & 1.195 & 5.611 & - & 8.901 \\
\hline 1980 & 4.170 & 5.510 & - & 96 & 41 & - & - & 5.893 & 14.287 & 274 & 30.271 \\
\hline 1985 & 4.479 & 10.061 & 258 & 311 & 195 & ${ }^{\mathrm{a}} 225$ & ${ }^{\mathrm{a}} 153$ & 13.551 & 16.125 & ${ }^{\mathrm{a}} 595$ & 44.980 \\
\hline 1990 & - & 12.271 & 296 & 432 & 145 & 393 & 358 & 28.610 & 30.355 & ${ }^{\mathrm{b}} 1.276$ & 72.860 \\
\hline 1995 & - & 20.902 & 563 & 646 & 261 & - & 833 & 52.103 & 37.594 & 2.680 & 115.582 \\
\hline 2000 & - & 39.823 & 1.785 & 1.980 & 1.189 & - & 2.414 & 120.873 & 91.738 & 5.859 & 265.661
\end{tabular}

\footnotetext{
${ }^{\mathrm{a}}$ Datos correspondientes a 1986

${ }^{\mathrm{b}}$ Datos correspondientes a 1991
}

Fuente: Elaboración propia con datos de Anuario Estadístico Cajas Rurales y Cooperativas de Crédito (1980), Anuario de las Cooperativas de Crédito y Cajas Rurales (1985), Anuario Cajas Rurales, Populares y Profesionales (1995 y 2000) y Titos Martínez (2003), cuadro 4.03, pp. 280-281. 


\section{ANEXO 3 NÚMERO DE OFICINAS DE LAS CAJAS RURALES ANDALUZAS (1975-2000)}

\section{ANDALUCÍA ORIENTAL}

\begin{tabular}{cccccccc}
\hline Año & $\begin{array}{c}\text { C.R. } \\
\text { Prov. } \\
\text { Almería }\end{array}$ & Cajamar & $\begin{array}{c}\text { C.R. } \\
\text { Prov. } \\
\text { Granada }\end{array}$ & $\begin{array}{c}\text { C.R. } \\
\text { Prov. } \\
\text { Jaén }\end{array}$ & $\begin{array}{c}\text { C.R. San José } \\
\text { (Torredonjimeno) }\end{array}$ & $\begin{array}{c}\text { C.R. } \\
\text { Prov. } \\
\text { Málaga }\end{array}$ & $\begin{array}{c}\text { Total } \\
\text { Andalucía } \\
\text { Oriental }\end{array}$ \\
\hline & & & & & & & \\
1975 & 59 & - & 71 & 89 & 1 & 57 & 277 \\
1980 & 76 & - & 84 & 89 & 1 & 58 & 308 \\
1985 & 98 & - & 100 & 89 & 1 & 65 & 353 \\
1990 & 143 & - & 99 & 91 & - & 78 & 411 \\
1995 & 225 & - & 116 & 106 & - & 86 & 533 \\
2000 & - & 567 & 140 & 124 & - & - & 831 \\
\hline
\end{tabular}

\section{ANDALUCÍA OCCIDENTAL}

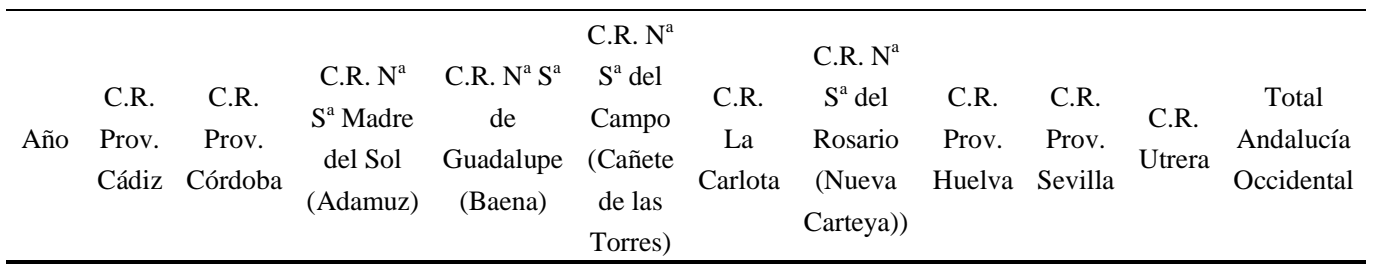

\begin{tabular}{rrrrrrrrrrrr}
1975 & 23 & 33 & 1 & 1 & 1 & 1 & 1 & 63 & 108 & 1 & 233 \\
1980 & 33 & 45 & 1 & 1 & 1 & 1 & 1 & 72 & 106 & 3 & 264 \\
1985 & 39 & 50 & 1 & 1 & 1 & 1 & 1 & 73 & 109 & 2 & 278 \\
1990 & - & 59 & 1 & 1 & 1 & 1 & 1 & 78 & 119 & 1 & 262 \\
1995 & - & 70 & 1 & 1 & 1 & - & 1 & 83 & 130 & 5 & 292 \\
2000 & - & 81 & 1 & 1 & 1 & - & 1 & 105 & 131 & 5 & 326 \\
\hline
\end{tabular}

Fuente: Elaboración propia con datos de Anuario Estadístico Cajas Rurales y Cooperativas de Crédito (1980), Anuario de las Cooperativas de Crédito y Cajas Rurales (1985), Anuario Cajas Rurales, Populares y Profesionales (1995 y 2000) y Titos Martínez (2003), cuadro 4.09 , pp. 292-293. 